\title{
Einstein-Ordered Weighted Geometric Operator for Pythagorean Fuzzy Soft Set with Its Application to Solve MAGDM Problem
}

\author{
Rana Muhammad Zulqarnain $\mathbb{D}^{1},{ }^{1}$ Imran Siddique, ${ }^{2}$ and Salwa EI-Morsy $\mathbb{D}^{3,4}$ \\ ${ }^{1}$ Department of Mathematics, School of Science, University of Management and Technology, Sialkot Campus, Lahore, \\ Punjab 54770, Pakistan \\ ${ }^{2}$ Department of Mathematics, School of Science, University of Management and Technology, Lahore 54770, Pakistan \\ ${ }^{3}$ Basic Science Department, Nile Higher Institute for Engineering and Technology, Mansoura, Egypt \\ ${ }^{4}$ Department of Mathematics, College of Science and Arts, Qassim University, Al-Badaya, Saudi Arabia
}

Correspondence should be addressed to Rana Muhammad Zulqarnain; ranazulqarnain7777@gmail.com and Salwa EI-Morsy; s.elmorsy@qu.edu.sa

Received 6 November 2021; Revised 26 November 2021; Accepted 6 December 2021; Published 24 January 2022

Academic Editor: Ewa Rak

Copyright (c) 2022 Rana Muhammad Zulqarnain et al. This is an open access article distributed under the Creative Commons Attribution License, which permits unrestricted use, distribution, and reproduction in any medium, provided the original work is properly cited.

\begin{abstract}
The Pythagorean fuzzy soft set (PFSS) is the most influential and operative tool for maneuvering compared to the Pythagorean fuzzy set (PFS), which can accommodate the parameterization of alternatives. It is also a generalized form of intuitionistic fuzzy soft sets (IFSS), which delivers healthier and more exact valuations in the decision-making (DM) procedure. The primary purpose is to extend and propose ideas related to Einstein's ordered weighted geometric aggregation operator from fuzzy structure to PFSS structure. The core objective of this work is to present a PFSS aggregation operator, such as the Pythagorean fuzzy soft Einsteinordered weighted geometric (PFSEOWG) operator. In addition, the basic properties of the proposed operator are introduced, such as idempotency, boundedness, and homogeneity. Moreover, a DM method based on a developed operator has been presented to solve the multiattribute group decision-making (MAGDM) problem. A real-life application of the anticipated method has been offered for a capitalist to choose the most delicate business to finance his money. Finally, a brief comparative analysis with some current methods demonstrates the proposed approach's effectiveness and reliability.
\end{abstract}

\section{Introduction}

MAGDM is considered the most appropriate technique to find the most acceptable alternative from all possible alternatives, following standards or attributes. Traditionally, it is assumed that all information for accessing options based on features and their corresponding weights are expressed in precise numbers. On the other hand, most decisions are made when goals and constraints are usually uncertain or unclear in real life. Zaheh [1] introduced the fuzzy set (FS) model to cope with the specified scenario, making progress in multiple scientific and technical fields. In traditional set theory, the elements of a set can be 0 or 1 , but in FS, the degree of membership ranges from 0 to 1 . Atanassov [2] extended the perception of FS and developed the notion of the intuitionistic fuzzy set (IFS), which deals with the uncertainty considering the membership (MG) and nonmembership (NMG) grades. Xu [3] protracted the IFS and introduced novel aggregation operators (AOs) for IFS. Wang and Liu [4] proposed some AOs for IFS based on Einstein operations and established a multiple attribute decision-making (MADM) approach using their presented operators. Atanassov [5] established the notion of intervalvalued IFS and discussed some essential operations with their properties.

IFS is an influential idea, and various scholars have considered it since its development. However, the leading concept of IFS has some shortcomings; for example, if the sum of membership and nonmembership degree is 1 , then IFS cannot deal with such scenarios. To overcome such 
complications, Yager [6] introduced the PFS, the most generalized form of IFS. Rehman et al. [7] proposed some AOs for PFS and discussed its properties. They also planned a DM approach using their developed AOs. Rehman et al. [8] offered a Pythagorean fuzzy ordered weighted geometric AO with necessary possessions. Wang and $\mathrm{Li}$ [9] introduced a MADM approach for PFS using Bonferroni mean AOs. Garg [10] established some novel AOs for PFS, considering the interaction based on Einstein operations.

The abovementioned models and their conforming DM approaches have been familiar and used by specialists in numerous areas. But these models cannot accommodate the parameterized values of the alternatives. Molodtsov [11] developed the concept of soft sets (SSs) and discussed some fundamental operations with their desirable properties. Maji et al. [12] prolonged the notion of SS and stated various elementary operations with its features and utilized it to solve DM complications [13]. Maji et al. [14] combined SS and IFS to grow IFSS and introduced necessary operations with their properties. Zulqarnain et al. [15] introduced the technique for order of preference by similarity to ideal solution (TOPSIS) procedure for interval-valued IFSS employing correlation coefficient (CC). Zulqarnain et al. [16] proposed the robust AOs for the intuitionistic fuzzy hypersoft set. They also constructed an MCDM model using their developed operators to solve DM issues. Zulqarnain et al. [17] proposed the TOPSIS method for intuitionistic fuzzy hypersoft set based on CC to resolve MADM problems. Garg and Arora [18] prolonged the IFSS and projected the generalized AOs for the IFSS.

Several scholars protracted SS ideas by engaging the crucial sorting of FSS. Peng et al. [19] progressed the condition of $M G+N M G \leq 1$ to $M^{2}+\mathrm{NMG}^{2} \leq 1$ of IFSS and established the PFSS with basic operations and possessions. Athira et al. [20] planned the entropy measure for PFSS. Siddique et al. [21] proposed some novel operations for PFSS and established a DM process based on a score matrix. Naeem et al. [22] stretched the concept of PFSS to linguistic PFSS and presented some necessary operations with their possessions. Riaz et al. [23] protracted the PFSS to $m$ polar PFSS and developed the TOPSIS technique to resolve the multicriteria group decision-making (MCGDM) problem. Zulqarnain et al. [24] introduced the Pythagorean fuzzy soft Einstein-ordered weighted average operator of PFSS and established the DM technique based on the operator developed by them. Zulqarnain et al. [25] introduced some novel operational laws for PFSS and settled some AOs for PFSS. Zulqarnain et al. [26] developed the TOPSIS method for PFSS based on CC and used their planned approach to resolve the MADM problem. Zulqarnain et al. [27] offered some novel operational laws for PFSS considering the interaction and developed the interaction AOs for PFSS. They also presented an MCDM technique using their proposed interactive AOs. Garg [28, 29] introduced several Einstein AOs under the PFS environment and established the DM techniques based on settled operators to resolve complex difficulties. The existing Einstein AOs and Einstein-weighted ordered AOs are just a weighted Pythagorean fuzzy argument. These PFS Einstein AOs cannot accommodate the parameterized values of alternatives. To overcome the above shortcomings, we focus on developing some novel Einstein AOs for PFSS.

To solve these shortcomings, we indicated the finest alternate with PFSS. Pythagorean fuzzy soft numbers support to conform with inexact statistics in the difficulties of everyday life. In this study, the PFSS operator and steering mechanism of PFSEOWG are based on the assumption of Pythagorean fuzzy soft number (PFSN). Therefore, compared to IFSS, IFS, and FS, it is better to maintain inaccurate and imprecise information flexibility. The core objective of this article is to focus on the development of the new AO for PFSS. It is expected that the operational laws of the proposed operations will be followed to solve the DM problem, and numerical example will be used to prove the effectiveness of the introduced DM method. The main benefit of the proposed operator is that the proposed operator can reduce the number of IFSS and fuzzy soft set (FSS) operators under certain confidence limits. The rest of the research is ordered as follows: Section 2 discusses fundamental concepts such as FS, IFS, PFS, SS, FSS, IFSS, and PFSS. In Section 3, we settled the PFSEOWG operator. Section 3 also discusses some desirable properties of the suggested operator. Section 4 develops the MAGDM method based on the proposed operator and provides a numerical example for selecting the most suitable vehicle. In Section 5, a comparison with some popular methods has been given. Section 6 gives the conclusion.

\section{Preliminaries}

This section contains some basic definitions, such as SS, IFS, PFS, IFSS, and PFSS, which will form the following manuscript's structure.

Definition 1 (see [11]). Let $X$ be a universal set and $\mathbb{N}=$ $\left\{t_{1}, t_{2}, t_{3}, \ldots, t_{m}\right\}$ be the set of attributes and $\Omega$ is a mapping such as $\Omega: \mathbb{N} \longrightarrow K^{X}$, where $K^{X}$ represents the subsets collection of $X$. Then, $(\Omega, \mathbb{N})$ is called a SS over $X$.

Definition 2 (see [30]). Let $X$ be a collection of substances, then a PFS $A$ over $X$ is defined as

$$
A=\left\{\left(t, \mathfrak{a}_{A}(t), \mathfrak{b}_{A}(t)\right) \mid t \in X\right\},
$$

where $\mathfrak{a}_{A}(t), \mathfrak{b}_{A}(t): X \longrightarrow[0,1]$ represents the $M G$ and NMG functions, respectively. Furthermore, $0 \leq \mathfrak{a}_{A}$ $(t)^{2}+\mathfrak{b}_{A}(t)^{2} \leq 1$ and $I=1-\mathfrak{a}(t)^{2}-\mathfrak{b}_{A}(t)^{2}$ is called degree of indeterminacy.

From the above definition, we can see that the only difference lies in the condition, that is, in IFS, what we deal with is the state $0 \leq \mathfrak{a}_{A}(t)+\mathfrak{b}_{A}(t) \leq 1$ and $I=1-$ $\mathfrak{a}_{A}(t)-\mathfrak{b}_{A}(t)$, whereas in PFS, we have condition $0 \leq \mathfrak{a}_{A}(t)^{2}+\mathfrak{b}_{A}(t)^{2} \leq 1$ and $I=1-\mathfrak{a}_{A}(t)^{2}-\mathfrak{b}_{A}(t)^{2}$.

Definition 3 (see [14]). Let $X$ be a universal set and $\mathbb{N}$ be set of attributes and $\Omega$ is a mapping such as $\Omega: \mathbb{N} \longrightarrow \mathrm{IK}^{X}$, where $\mathrm{IK}^{X}$ is a collection of intuitionistic fuzzy subsets. Then, $(\Omega, \mathbb{N})$ is named an IFSS over $X$. 


$$
(\Omega, A)=\left\{t,\left(\mathfrak{a}_{A}(t), \mathfrak{b}_{A}(t)\right) \mid t \in A\right\},
$$

where $\mathfrak{a}_{A}(t), \mathfrak{b}_{A}(t): A \longrightarrow[0,1]$ are MG and NMG functions respectively with $0 \leq \mathfrak{a}_{A}(t)+\mathfrak{b}_{A}(t) \leq 1$ and $A \subset \mathbb{N}$.

Definition 4 (see [14]). Let $(\Omega, A)$ and $(\Omega, B)$ be two IFSS. Then, some basic operations for IFSS are defined as follows:

(1) $(\Omega, A)$ is said to be an intuitionistic fuzzy soft subset of $(\Omega, B)$. If $A \subset B$ and $\mathfrak{a}_{A}(t) \leq \mathfrak{a}_{B}(t), \mathfrak{b}_{A}(t) \geq \mathfrak{b}_{B}(t)$ for all $t \in A$.

(2) Complement of $(\Omega, A)$ is denoted by $\left(\Omega, A^{c}\right)$ and is defined as

$$
\left(\Omega, A^{c}\right)=\left\{t,\left(\mathfrak{b}_{A}(t), \mathfrak{a}_{A}(t)\right) \mid t \in A\right\} .
$$

(3) Union of two IFSS is defined as follows:

$$
(\Omega, A) \cup(\Omega, B)=\left\{t, \max \left(\mathfrak{a}_{A}(t), \mathfrak{a}_{B}(t)\right) \mid \forall t \in A\right\} .
$$

(4) The intersection of $(\Omega, A)$ and $(\Omega, B)$ can be defined as follows:

$$
(\Omega, A) \cap(\Omega, B)=\left\{t, \min \left(\mathfrak{a}_{A}(t), \mathfrak{a}_{B}(t)\right) \mid \forall t \in A\right\} .
$$

Definition 5 (see [19]). Let $X$ be a universal set and $\mathbb{N}$ be set of attributes and $\Omega$ be a mapping such as $\Omega: \mathbb{N} \longrightarrow \wp K^{X}$, where $\wp K^{X}$ is a collection of Pythagorean fuzzy subsets. Then, $(\Omega, \mathbb{N})$ is called a PFSS over $X$.

$$
(\Omega, A)=\left\{t,\left(\mathfrak{a}_{A}(t), \mathfrak{b}_{A}(t)\right) \mid t \in A\right\} .
$$

where $\mathfrak{a}_{A}(t), \mathfrak{b}_{A}(t): A \longrightarrow[0,1]$ represents the $M G$ and NMG functions, respectively, with $0 \leq \mathfrak{a}_{A}(t)^{2}+\mathfrak{b}_{A}(t)^{2} \leq 1$, degree of independency $\mathfrak{I}=1-\mathfrak{a}_{A}(t)^{2}-\mathfrak{b}_{A}(t)^{2}$, and $A \subset \mathbb{N}$.

The PFSN can be expressed as $\mathscr{H}_{i j}=\mathfrak{a}_{i j}, \mathfrak{b}_{i j}$ for readers' convenience. Zulqarnain et al. [25] presented the score and accuracy functions for PFSN such as

$$
S\left(H_{i j}\right)=\mathfrak{a}_{i j}^{2}-\mathfrak{b}_{i j}^{2}
$$

where $S\left(H_{i j}\right) \in[-1,1]$. Sometimes, score function cannot differentiate the PFSNs. For example, let $H_{11}=0.3162,0.4472$ and $H_{12}=0.5477,0.6324$, then equation (7); we have $S\left(H_{11}\right)=-0.1$ and $S\left(H_{12}\right)=-0.1$. So, it is difficult to decide which alternative is most suitable in this case. An accuracy function has been developed to overcome the limitations mentioned above.

$$
A\left(H_{i j}\right)=\mathfrak{a}_{i j}^{2}+\mathfrak{b}_{i j}^{2},
$$

where $A\left(\mathscr{H}_{i j}\right) \in[-1,1]$. The following comparison laws have been presented for PFSNs.

(1) If $S\left(H_{i j}\right)>S\left(R_{i j}\right)$, then $H_{i j}>H_{i j}$.

(2) If $S\left(H_{i j}\right)=S\left(R_{i j}\right)$, then

(i) If $A\left(H_{i j}\right)>A\left(R_{i j}\right)$, then $H_{i j}>R_{i j}$.

(ii) If $A\left(H_{i j}\right)=A\left(R_{i j}\right)$, then $H_{i j}=R_{i j}$.

\section{Einstein-Ordered Weighted Geometric Operator for Pythagorean Fuzzy Soft Set}

The subsequent section will develop the Einstein-ordered weighted geometric operator for PFSS with some fundamental properties.

Definition 6. Let $H_{i j}=\left(\mathfrak{a}_{i j}, \mathfrak{b}_{i j}\right)$ be a collection of PFSNs, where $(i=1,2, \ldots, n)$ and $(j=1,2, \ldots, m)$, then the Pythagorean fuzzy soft Einstein-ordered weighted geometric (PFSEOWG) operator is defined as

$$
\operatorname{PFSEOWG}\left(H_{11}, H_{12}, \ldots, H_{n m}\right)=\oplus_{j=1}^{m} \mathscr{J}_{j}\left(\oplus_{i=1}^{n} \mathcal{O}_{i} H_{\mathfrak{r}(i) \mathfrak{I}(j)}\right) \text {, }
$$

where $\mathscr{O}_{i}$ and $\mathscr{J}_{j}$ represent the weight vectors such that $\mathcal{O}_{i}>0, \sum_{i=1}^{n} \mathcal{O}_{i}=1$ and $\mathscr{J}_{j}>0$, and $\sum_{j=1}^{n} \mathscr{J}_{j}=1$ and $\mathfrak{r}, \mathfrak{s}$ are permutations of $(i=1,2, \ldots, n)$ and $(j=1,2, \ldots, m)$ such that $H_{\mathfrak{r}(i-1) j} \geq H_{\mathfrak{r}(i) j}$ and $H_{i \mathfrak{S}(j-1)} \geq H_{i \mathfrak{S}(j)} \forall i, j$.

Theorem 1. Let $H_{i j}=\left(a_{i j}, b_{i j}\right)$ be a collection of PFSNs, where $(i=1,2, \ldots, n)$ and $(j=1,2, \ldots, m)$, then the aggregated value obtained by equation (9) is given as

$$
\begin{aligned}
& \operatorname{PFSEOWG}\left(H_{11}, H_{12}, \ldots, H_{\mathrm{nm}}\right)=\oplus_{j=1}^{m} \mathscr{J}_{j}\left(\oplus_{i=1}^{n} \mathcal{O}_{i} H_{\mathfrak{r}(i) \mathfrak{z}(j)}\right) \\
& =\left\langle\frac{\sqrt{2 \prod_{j=1}^{m}\left(\prod_{i=1}^{n}\left(\mathfrak{a}_{\mathfrak{r}(i) \mathfrak{B}(j)}^{2}\right)^{\mathcal{G}_{i}}\right)^{\mathcal{F}_{j}}}}{\sqrt{\prod_{j=1}^{m}\left(\prod_{i=1}^{n}\left(2-\mathfrak{a}_{\mathfrak{r}(i) \mathfrak{B}(j)}^{2}\right)^{\mathcal{O}_{i}}\right)^{\mathcal{F}_{j}}+\prod_{j=1}^{m}\left(\prod_{i=1}^{n}\left(\mathfrak{a}_{\mathfrak{r}(i) \mathfrak{B}(j)}^{2}\right)^{\mathcal{O}_{i}}\right)^{\mathcal{F}_{j}}}},\right. \\
& \left.\cdot \frac{\sqrt{\prod_{j=1}^{m}\left(\prod_{i=1}^{n}\left(1+\mathfrak{b}_{\mathfrak{r}(i) \mathfrak{s}(j)}^{2}\right)^{\mathcal{G}_{i}}\right)^{\mathcal{F}_{j}}-\prod_{j=1}^{m}\left(\prod_{i=1}^{n}\left(1-\mathfrak{b}_{\mathfrak{r}(i) \mathfrak{b}(j)}^{2}\right)^{\mathcal{G}_{i}}\right)^{\mathcal{F}_{j}}}}{\sqrt{\prod_{j=1}^{m}\left(\prod_{i=1}^{n}\left(1+\mathfrak{b}_{\mathfrak{r}(i) \mathfrak{s}(j)}^{2}\right)^{\mathcal{G}_{i}}\right)^{\mathcal{F}_{j}}+\prod_{j=1}^{m}\left(\prod_{i=1}^{n}\left(1-\mathfrak{b}_{\mathfrak{r}(i) \mathfrak{s}(j)}^{2}\right)^{\mathcal{G}_{i}}\right)^{\mathcal{F}_{j}}}}\right\rangle
\end{aligned}
$$

where $\mathcal{O}_{i}$ and $\mathscr{J}_{j}$ represent the weight vectors such that $\mathscr{O}_{i}>0$, $\sum_{i=1}^{n} \mathcal{O}_{i}=1$ and $\mathscr{J}_{j}>0$, and $\sum_{j=1}^{n} \mathscr{J}_{j}=1$ and $\mathfrak{x}, \mathfrak{g}$ are permutations of $(i=1,2, \ldots, n)$ and $(j=1,2, \ldots, m)$ such that $H_{\mathfrak{r}(i-1) j} \geq H_{\mathfrak{r}(i) j}$ and $H_{i \mathfrak{S}(j-1)} \geq H_{i \mathfrak{S}(j)} \forall i, j$. 
Proof. We will prove it by using mathematical induction.

For $n=1$, we get $\mathcal{O}_{i}=1$.

$\operatorname{PFSEOWG}\left(H_{11}, H_{12}, \ldots, H_{n m}\right)=\oplus_{j=1}^{m} \mathscr{J}_{j} H_{\mathfrak{r}(1) \mathfrak{g}(j)}$

$$
\begin{aligned}
& =\left\langle\frac{\sqrt{2 \prod_{j=1}^{m}\left(\mathfrak{a}_{\mathfrak{r}(1) \mathfrak{z}(j)}^{2}\right)^{\mathcal{F}_{j}}}}{\sqrt{\prod_{j=1}^{m}\left(2-\mathfrak{a}_{\mathfrak{r}(1) \mathfrak{I}(j)}^{2}\right)^{\mathcal{F}_{j}}+\prod_{j=1}^{m}\left(\mathfrak{a}_{\mathfrak{r}(1) \mathfrak{z}(j)}^{2}\right)^{\mathcal{F}_{j}}}},\right. \\
& \left.\cdot \frac{\sqrt{\prod_{j=1}^{m}\left(1+\mathfrak{b}_{\mathfrak{r}(1) \mathfrak{z}(j)}^{2}\right)^{\mathcal{F}_{j}}-\prod_{j=1}^{m}\left(1-\mathfrak{b}_{\mathfrak{r}(1) \mathfrak{z}(j)}^{2}\right)^{\mathscr{F}_{j}}}}{\sqrt{\prod_{j=1}^{m}\left(1+\mathfrak{b}_{\mathfrak{r}(1) \mathfrak{z}(j)}^{2}\right)^{\mathcal{F}_{j}}+\prod_{j=1}^{m}\left(1-\mathfrak{b}_{\mathfrak{r}(1) \mathfrak{s}(j)}^{2}\right)^{\mathcal{F}_{j}}}}\right\rangle \\
& =\left\langle\frac{\sqrt{2 \prod_{j=1}^{m}\left(\prod_{j=1}^{m}\left(\mathfrak{a}_{\mathfrak{r}(i) \mathfrak{S}(j)}^{2}\right)^{\mathcal{G}_{i}}\right)^{\mathcal{F}_{j}}}}{\sqrt{\prod_{j=1}^{m}\left(\prod_{j=1}^{m}\left(2-\mathfrak{a}_{\mathfrak{r}(i) \mathfrak{I}(j)}^{2}\right)^{\mathcal{G}_{i}}\right)^{\mathcal{F}_{j}}+\prod_{j=1}^{m}\left(\prod_{j=1}^{m}\left(\mathfrak{a}_{\mathfrak{r}(i) \mathfrak{I}(j)}^{2}\right)^{\mathcal{G}_{i}}\right)^{\mathcal{F}_{j}}}},\right. \\
& \left.\cdot \frac{\sqrt{\prod_{j=1}^{m}\left(\prod_{j=1}^{m}\left(1+\mathfrak{b}_{\mathfrak{r}(i) \mathfrak{B}(j)}^{2}\right)^{\mathcal{O}_{i}}\right)^{\mathcal{F}_{j}}-\prod_{j=1}^{m}\left(\prod_{j=1}^{m}\left(1-\mathfrak{b}_{\mathfrak{r}(i) \mathfrak{L}(j)}^{2}\right)^{\mathcal{G}_{i}}\right)^{\mathcal{F}_{j}}}}{\sqrt{\prod_{j=1}^{m}\left(\prod_{j=1}^{m}\left(1+\mathfrak{b}_{\mathfrak{r}(i) \mathfrak{S}(j)}^{2}\right)^{\mathcal{O}_{i}}\right)^{\mathcal{F}_{j}}+\prod_{j=1}^{m}\left(\prod_{j=1}^{m}\left(1-\mathfrak{b}_{\mathfrak{r}(i) \mathfrak{I}(j)}^{2}\right)^{\mathcal{G}_{i}}\right)^{\mathcal{F}_{j}}}}\right\rangle .
\end{aligned}
$$

For $m=1$, we get $\mathscr{J}_{j}=1$.

$$
\begin{aligned}
& \operatorname{PFSEOWG}\left(H_{11}, H_{12}, \ldots, H_{n m}\right)=\oplus_{i=1}^{n} \mathcal{O}_{i} H_{\mathfrak{r}(i) \mathfrak{g}(1)} \\
& =\left\langle\frac{\sqrt{2 \prod_{i=1}^{n}\left(\mathfrak{a}_{\mathfrak{r}(i) \mathfrak{s}(1)}^{2}\right)^{\sigma_{i}}}}{\sqrt{\prod_{i=1}^{n}\left(2-\mathfrak{a}_{\mathbf{r}(i) \mathfrak{s}(j)}^{2}\right)^{\sigma_{i}}+\prod_{i=1}^{n}\left(\mathfrak{a}_{\mathfrak{r}(i) \mathfrak{s}(1)}^{2}\right)^{\sigma_{i}}}},\right. \\
& \left.\cdot \frac{\sqrt{\prod_{i=1}^{n}\left(1+\mathfrak{b}_{\mathfrak{r}(i) \mathfrak{s}(1)}^{2}\right)^{\sigma_{i}}-\prod_{i=1}^{n}\left(1-\mathfrak{b}_{\mathfrak{r}(i) \mathfrak{s}(1)}^{2}\right)^{\sigma_{i}}}}{\sqrt{\prod_{i=1}^{n}\left(1+\mathfrak{b}_{\mathfrak{r}(i) \mathfrak{s}(1)}^{2}\right)^{\sigma_{i}}+\prod_{i=1}^{n}\left(1-\mathfrak{b}_{\mathfrak{r}(i) \mathfrak{s}(1)}^{2}\right)^{\sigma_{i}}}}\right\rangle \\
& =\left\langle\frac{\sqrt{2 \prod_{i=1}^{n}\left(\prod_{i=1}^{n}\left(\mathfrak{a}_{\mathfrak{r}(i) \mathfrak{s}(j)}^{2}\right)^{\mathscr{G}_{i}}\right)^{\mathcal{F}_{j}}}}{\sqrt{\prod_{i=1}^{n}\left(\prod_{i=1}^{n}\left(2-\mathfrak{a}_{\mathfrak{r}(i) \mathfrak{s}(j)}^{2}\right)^{\mathscr{G}_{i}}\right)^{\mathcal{F}_{j}}+\prod_{i=1}^{n}\left(\prod_{i=1}^{n}\left(\mathfrak{a}_{\mathfrak{r}(i) \mathfrak{s}(j)}^{2}\right)^{\mathscr{G}_{i}}\right)^{\mathscr{G}_{j}}}},\right.
\end{aligned}
$$

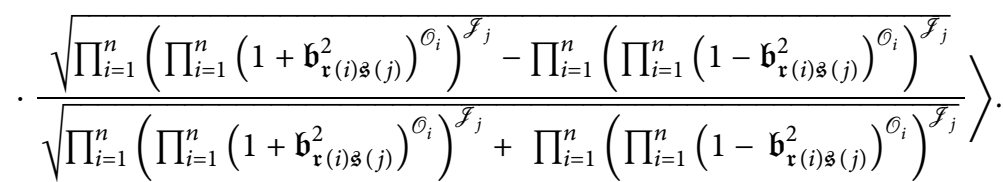

So, equation (9) is true for $n=1$ and $m=1$. 
Suppose that equation (9) holds for $n=\delta_{2}, m=\delta_{1}+1$ and for $n=\delta_{2}+1, m=\delta_{1}$

$$
\begin{aligned}
& \oplus_{j=1}^{\delta_{1}+1} \mathscr{J}_{j}\left(\oplus_{i=1}^{\delta_{2}} \mathcal{O}_{i} H_{\mathfrak{r}(i) \mathfrak{Z}(j)}\right) \\
& =\left\langle\frac{\sqrt{2 \prod_{j=1}^{\delta_{1}+1}\left(\prod_{i=1}^{\delta_{2}}\left(\mathfrak{a}_{\mathfrak{r}(i) \mathfrak{g}(j)}^{2}\right)^{\mathcal{G}_{i}}\right)^{\mathcal{F}_{j}}}}{\sqrt{\prod_{j=1}^{\delta_{1}+1}\left(\prod_{i=1}^{\delta_{2}}\left(2-\mathfrak{a}_{\mathfrak{r}(i) \mathfrak{I}(j)}^{2}\right)^{\mathcal{O}_{i}}\right)^{\mathcal{F}_{j}}+\prod_{j=1}^{\delta_{1}+1}\left(\prod_{i=1}^{\delta_{2}}\left(\mathfrak{a}_{\mathfrak{r}(i) \mathfrak{I}(j)}^{2}\right)^{\mathcal{G}_{i}}\right)^{\mathcal{F}_{j}}}},\right. \\
& \left.\cdot \frac{\sqrt{\prod_{j=1}^{\delta_{1}+1}\left(\prod_{i=1}^{\delta_{2}}\left(1+\mathfrak{b}_{\mathfrak{r}(i) \mathfrak{b}(j)}^{2}\right)^{\mathcal{G}_{i}}\right)^{\mathcal{F}_{j}}-\prod_{j=1}^{\delta_{1}+1}\left(\prod_{i=1}^{\delta_{2}}\left(1-\mathfrak{b}_{\mathfrak{r}(i) \mathfrak{b}(j)}^{2}\right)^{\mathcal{G}_{i}}\right)^{\mathcal{F}_{j}}}}{\sqrt{\prod_{j=1}^{\delta_{1}+1}\left(\prod_{i=1}^{\delta_{2}}\left(1+\mathfrak{b}_{\mathfrak{r}(i) \mathfrak{s}(j)}^{2}\right)^{\mathcal{G}_{i}}\right)^{\mathcal{F}_{j}}+\prod_{j=1}^{\delta_{1}+1}\left(\prod_{i=1}^{\delta_{2}}\left(1-\mathfrak{b}_{\mathfrak{r}(i) \mathfrak{s}(j)}^{2}\right)^{\mathcal{G}_{i}}\right)^{\mathcal{F}_{j}}}}\right\rangle \\
& \oplus_{j=1}^{\delta_{1}} \mathscr{J}_{j}\left(\oplus_{i=1}^{\delta_{2}+1} \mathcal{O}_{i} H_{\mathfrak{r}(i) \mathfrak{z}(j)}\right) \\
& =\left\langle\frac{\sqrt{2 \prod_{j=1}^{\delta_{1}}\left(\prod_{i=1}^{\delta_{2}+1}\left(\mathfrak{a}_{\mathfrak{r}(i) \mathfrak{I}(j)}^{2}\right)^{\mathcal{O}_{i}}\right)^{\mathcal{F}_{j}}}}{\sqrt{\prod_{j=1}^{\delta_{1}}\left(\prod_{i=1}^{\delta_{2}+1}\left(2-\mathfrak{a}_{\mathfrak{r}(i) \mathfrak{z}(j)}^{2}\right)^{\mathcal{O}_{i}}\right)^{\mathcal{F}_{j}}+\prod_{j=1}^{\delta_{1}}\left(\prod_{i=1}^{\delta_{2}+1}\left(\mathfrak{a}_{\mathfrak{r}(i) \mathfrak{I}(j)}^{2}\right)^{\mathcal{O}_{i}}\right)^{\mathcal{F}_{j}}}},\right. \\
& \left.\cdot \frac{\sqrt{\prod_{j=1}^{\delta_{1}}\left(\prod_{i=1}^{\delta_{2}+1}\left(1+\mathfrak{b}_{\mathfrak{r}(i) \mathfrak{I}(j)}^{2}\right)^{\mathcal{G}_{i}}\right)^{\mathcal{F}_{j}}-\prod_{j=1}^{\delta_{1}}\left(\prod_{i=1}^{\delta_{2}+1}\left(1-\mathfrak{b}_{\mathfrak{r}(i) \mathfrak{z}(j)}^{2}\right)^{\mathcal{G}_{i}}\right)^{\mathcal{F}_{j}}}}{\sqrt{\prod_{j=1}^{\delta_{1}}\left(\prod_{i=1}^{\delta_{2}+1}\left(1+\mathfrak{b}_{\mathfrak{r}(i) \mathfrak{I}(j)}^{2}\right)^{\mathcal{G}_{i}}\right)^{\mathcal{F}_{j}}+\prod_{j=1}^{\delta_{1}}\left(\prod_{i=1}^{\delta_{2}+1}\left(1-\mathfrak{b}_{\mathfrak{r}(i) \mathfrak{g}(j)}^{2}\right)^{\mathcal{G}_{i}}\right)^{\mathcal{F}_{j}}}}\right\rangle .
\end{aligned}
$$

Now, we prove equation (9) for $m=\delta_{1}+1$ and $n=\delta_{2}+1$

$$
\begin{aligned}
& \oplus_{j=1}^{\delta_{1}+1} \mathscr{J}_{j}\left(\oplus_{i=1}^{\delta_{2}+1} \mathcal{O}_{i} H_{\mathfrak{r}(i) \mathfrak{B}(j)}\right) \\
& =\left(\oplus_{j=1}^{\delta_{1}+1} \oplus_{i=1}^{\delta_{2}} \mathcal{O}_{i} \mathscr{J}_{j} \mathrm{H}_{\mathfrak{r}(i) \mathfrak{I}(j)}\right)\left(\oplus_{j=1}^{\delta_{1}+1} \mathscr{J}_{j} \mathcal{O}_{i+1} H_{\mathfrak{r}\left(\delta_{2}+1\right) \mathfrak{s}(j)}\right) \\
& =\left\langle\frac{\sqrt{2 \prod_{j=1}^{\delta_{1}+1}\left(\prod_{i=1}^{\delta_{2}}\left(\mathfrak{a}_{\mathfrak{r}(i) \mathfrak{I}(j)}^{2}\right)^{\mathcal{O}_{i}}\right)^{\mathcal{F}_{j}}}}{\sqrt{\prod_{j=1}^{\delta_{1}+1}\left(\prod_{i=1}^{\delta_{2}}\left(2-\mathfrak{a}_{\mathfrak{r}(i) \mathfrak{s}(j)}^{2}\right)^{\mathcal{O}_{i}}\right)^{\mathcal{F}_{j}}+\prod_{j=1}^{\delta_{1}+1}\left(\prod_{i=1}^{\delta_{2}}\left(\mathfrak{a}_{\mathfrak{r}(i) \mathfrak{I}(j)}^{2}\right)^{\mathcal{O}_{i}}\right)^{\mathcal{F}_{j}}}}\right. \\
& \oplus \frac{\sqrt{2 \prod_{j=1}^{\delta_{1}+1}\left(\left(\mathfrak{a}_{\mathfrak{r}\left(\delta_{2}+1\right) \mathfrak{s}(j)}^{2}\right)^{\mathcal{O}_{\delta_{2}+1}}\right)}}{\sqrt{\prod_{j=1}^{\delta_{1}+1}\left(\left(2-\mathfrak{a}_{\mathfrak{r}\left(\delta_{2}+1\right) \mathfrak{s}(j)}^{2}\right)^{\mathcal{G}_{\delta_{2}+1}}\right)^{\mathcal{F}_{j}}+\prod_{j=1}^{\delta_{1}+1}\left(\left(\mathfrak{a}_{\mathfrak{r}\left(\delta_{2}+1\right) \mathfrak{s}(j)}^{2}\right)^{\mathcal{G}_{\delta_{2}+1}}\right)^{\mathcal{J}_{j}}}}
\end{aligned}
$$




$$
\begin{aligned}
& =\frac{\sqrt{\prod_{j=1}^{\delta_{1}+1}\left(\prod_{i=1}^{\delta_{2}}\left(1+\mathfrak{b}_{\mathfrak{r}(i) \mathfrak{z}(j)}^{2}\right)^{\mathcal{O}_{i}}\right)^{\mathcal{F}_{j}}-\prod_{j=1}^{\delta_{1}+1}\left(\prod_{i=1}^{\delta_{2}}\left(1-\mathfrak{b}_{\mathfrak{r}(i) \mathfrak{s}(j)}^{2}\right)^{\mathcal{G}_{i}}\right)^{\mathcal{F}_{j}}}}{\sqrt{\prod_{j=1}^{\delta_{1}+1}\left(\prod_{i=1}^{\delta_{2}}\left(1+\mathfrak{b}_{\mathfrak{r}(i) \mathfrak{g}(j)}^{2}\right)^{\mathcal{G}_{i}}\right)^{\mathcal{F}_{j}}+\prod_{j=1}^{\delta_{1}+1}\left(\prod_{i=1}^{\delta_{2}}\left(1-\mathfrak{b}_{\mathfrak{r}(i) \mathfrak{z}(j)}^{2}\right)^{\mathcal{G}_{i}}\right)^{\mathcal{F}_{j}}}} \\
& \left.\oplus \frac{\sqrt{\prod_{j=1}^{\delta_{1}+1}\left(\left(1+\mathfrak{b}_{\mathfrak{r}\left(\delta_{2}+1\right) \mathfrak{s}(j)}^{2}\right)^{\mathcal{O}_{\delta_{2}+1}}\right)^{\mathcal{F}_{j}}-\prod_{j=1}^{\delta_{1}+1}\left(\left(1-\mathfrak{b}_{\mathfrak{r}\left(\delta_{2}+1\right) \mathfrak{s}(j)}^{2}\right)^{\mathcal{O}_{\delta_{2}+1}}\right)^{\mathcal{F}_{j}}}}{\sqrt{\prod_{j=1}^{\delta_{1}+1}\left(\left(1+\mathfrak{b}_{\mathfrak{r}\left(\delta_{2}+1\right) \mathfrak{s}(j)}^{2}\right)^{\mathcal{O}_{\delta_{2}+1}}\right)^{\mathcal{F}_{j}}+\prod_{j=1}^{\delta_{1}+1}\left(\left(1-\mathfrak{b}_{\mathfrak{r}\left(\delta_{2}+1\right) \mathfrak{s}(j)}^{2}\right)^{\mathcal{O}_{\delta_{2}+1}}\right)^{\mathcal{F}_{j}}}}\right\rangle \\
& =\frac{\sqrt{2 \prod_{j=1}^{\delta_{1}+1}\left(\prod_{i=1}^{\delta_{2}+1}\left(\mathfrak{a}_{\mathfrak{r}(i) \mathfrak{z}(j)}^{2}\right)^{\mathcal{G}_{i}}\right)^{\mathcal{F}_{j}}}}{\sqrt{\prod_{j=1}^{\delta_{1}+1}\left(\prod_{i=1}^{\delta_{2}+1}\left(2-\mathfrak{a}_{\mathfrak{r}(i) \mathfrak{s}(j)}^{2}\right)^{\mathcal{O}_{i}}\right)^{\mathcal{F}_{j}}+\prod_{j=1}^{\delta_{1}+1}\left(\prod_{i=1}^{\delta_{2}+1}\left(\mathfrak{a}_{\mathfrak{r}(i) \mathfrak{z}(j)}^{2}\right)^{\mathcal{O}_{i}}\right)^{\mathcal{F}_{j}}}}, \\
& \left.\frac{\sqrt{\prod_{j=1}^{\delta_{1}+1}\left(\prod_{i=1}^{\delta_{2}+1}\left(1+\mathfrak{b}_{\mathfrak{r}(i) \mathfrak{s}(j)}^{2}\right)^{\mathcal{G}_{i}}\right)^{\mathcal{F}_{j}}-\prod_{j=1}^{\delta_{1}+1}\left(\prod_{i=1}^{\delta_{2}+1}\left(1-\mathfrak{b}_{\mathfrak{r}(i) \mathfrak{g}(j)}^{2}\right)^{\mathcal{G}_{i}}\right)^{\mathcal{F}_{j}}}}{\sqrt{\prod_{j=1}^{\delta_{1}+1}\left(\prod_{i=1}^{\delta_{2}+1}\left(1+\mathfrak{b}_{\mathfrak{r}(i) \mathfrak{s}(j)}^{2}\right)^{\mathcal{G}_{i}}\right)^{\mathcal{F}_{j}}+\prod_{j=1}^{\delta_{1}+1}\left(\prod_{i=1}^{\delta_{2}+1}\left(1-\mathfrak{b}_{\mathfrak{r}(i) \mathfrak{z}(j)}^{2}\right)^{\mathcal{G}_{i}}\right)^{\mathcal{F}_{j}}}}\right\rangle \\
& =\oplus_{j=1}^{\delta_{1}+1} \mathscr{J}_{j}\left(\oplus_{i=1}^{\delta_{2}+1} \mathcal{O}_{i} \mathrm{H}_{\mathfrak{r}(i) \mathfrak{z}(j)}\right) .
\end{aligned}
$$

So, it is valid for $m=\delta_{1}+1$ and $n=\delta_{2}+1$.

Example 1. Let $R=\left\{R_{1}, R_{2}, R_{3}\right\}$ be a set of decision makers with weigh vector $\mathscr{O}_{i}=(0.1,0.3,0.3,0.3)^{T}$, who want to decide a bike under the set of attributes $A=\left\{A_{1}=\right.$ fuel milage, $A_{2}=$ speed per hour, $A_{3}=$ price, $A_{4}=$ comfort level, $A_{5}=$ design $\}$ with weight vector $\mathscr{J}_{j}=(0.2,0.2,0.2,0.4)^{T}$. Then, $\left(H_{4 \times 4}, A\right)=\left(\mathfrak{a}_{i j}, \mathfrak{b}_{i j}\right)_{4 \times 4}$ is given as

$$
\left(H_{4 \times 4}, A\right)=\left|\begin{array}{cccc}
(0.5,0.8) & (0.7,0.5) & (0.4,0.6) & (0.7,0.4) \\
(0.5,0.6) & (0.9,0.1) & (0.3,0.7) & (0.4,0.5) \\
(0.4,0.8) & (0.7,0.5) & (0.4,0.6) & (0.3,0.5) \\
(0.3,0.7) & (0.6,0.5) & (0.5,0.4) & (0.5,0.7)
\end{array}\right|
$$

First, we find the associated ordered position matrix by using the score function, which is

$$
\left(H_{4 \times 4}, A\right)=\left\lceil\begin{array}{llll}
(0.7,0.4) & (0.7,0.5) & (0.4,0.6) & (0.5,0.8) \\
(0.7,0.1) & (0.4,0.5) & (0.3,0.7) & (0.5,0.6) \\
(0.6,0.5) & (0.5,0.4) & (0.3,0.7) & (0.5,0.7)
\end{array}\right],
$$

as we know that

$$
\begin{aligned}
& \operatorname{PFSEOWG}\left(H_{11}, H_{12}, \ldots, H_{n m}\right) \\
& =\left\langle\frac{\sqrt{2 \prod_{j=1}^{m}\left(\prod_{i=1}^{n}\left(\mathfrak{a}_{\mathfrak{r}(i) \mathfrak{B}(j)}^{2}\right)^{\mathcal{G}_{i}}\right)^{\mathcal{F}_{j}}}}{\sqrt{\prod_{j=1}^{m}\left(\prod_{i=1}^{n}\left(2-\mathfrak{a}_{\mathfrak{r}(i) \mathfrak{g}(j)}^{2}\right)^{\mathcal{G}_{i}}\right)^{\mathcal{F}_{j}}+\prod_{j=1}^{m}\left(\prod_{i=1}^{n}\left(\mathfrak{a}_{\mathfrak{r}(i) \mathfrak{g}(j)}^{2}\right)^{\mathcal{G}_{i}}\right)^{\mathcal{F}_{j}}}},\right. \\
& \left.\frac{\sqrt{\prod_{j=1}^{m}\left(\prod_{i=1}^{n}\left(1+\mathfrak{b}_{\mathfrak{r}(i) \mathfrak{s}(j)}^{2}\right)^{\mathcal{G}_{i}}\right)^{\mathcal{F}_{j}}-\prod_{j=1}^{m}\left(\prod_{i=1}^{n}\left(1-\mathfrak{b}_{\mathfrak{r}(i) \mathfrak{s}(j)}^{2}\right)^{\mathcal{G}_{i}}\right)^{\mathcal{F}_{j}}}}{\sqrt{\prod_{j=1}^{m}\left(\prod_{i=1}^{n}\left(1+\mathfrak{b}_{\mathfrak{r}(i) \mathfrak{s}(j)}^{2}\right)^{\mathcal{G}_{i}}\right)^{\mathcal{F}_{j}}+\prod_{j=1}^{m}\left(\prod_{i=1}^{n}\left(1-\mathfrak{b}_{\mathfrak{r}(i) \mathfrak{s}(j)}^{2}\right)^{\mathcal{G}_{i}}\right)^{\mathcal{F}_{j}}}}\right\rangle,
\end{aligned}
$$$$
\operatorname{PFSEOWG}\left(H_{11}, H_{12}, \ldots, H_{44}\right)
$$ 


$$
\begin{aligned}
& =\left\langle\frac{\sqrt{2 \prod_{j=1}^{4}\left(\prod_{i=1}^{4}\left(\mathfrak{a}_{\mathfrak{r}(i) \mathfrak{s}(j)}^{2}\right)^{\mathcal{G}_{i}}\right)^{\mathcal{F}_{j}}}}{\sqrt{\prod_{j=1}^{4}\left(\prod_{i=1}^{4}\left(2-\mathfrak{a}_{\mathfrak{r}(i) \mathfrak{S}(j)}^{2}\right)^{\mathscr{G}_{i}}\right)^{\mathcal{F}_{j}}+\prod_{j=1}^{4}\left(\prod_{i=1}^{4}\left(\mathfrak{a}_{\mathfrak{r}(i) \mathfrak{s}(j)}^{2}\right)^{\mathcal{G}_{i}}\right)^{\mathcal{F}_{j}}}},\right.
\end{aligned}
$$

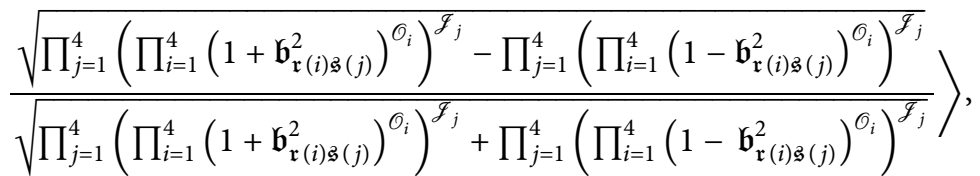

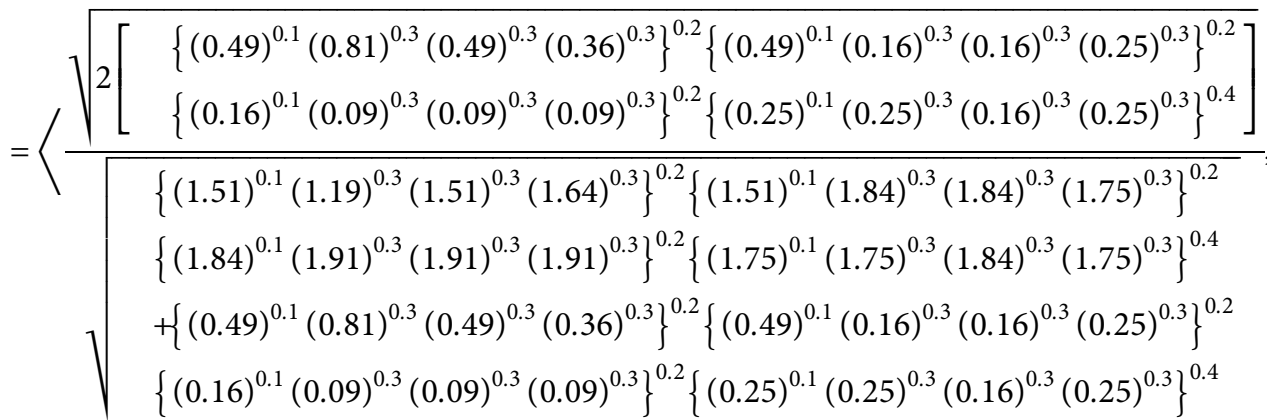

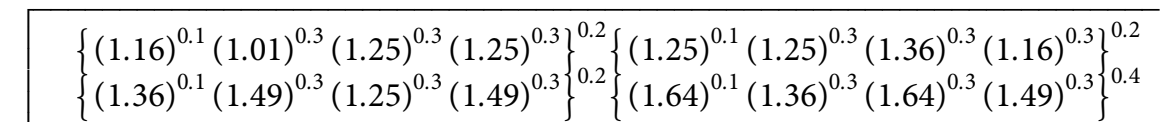

$$
\begin{aligned}
& \left.\frac{\sqrt{\left\{(0.84)^{0.1}(0.99)^{0.3}(0.75)^{0.3}(0.75)^{0.3}\right\}^{0.2}\left\{(0.75)^{0.1}(0.75)^{0.3}(0.64)^{0.3}(0.84)^{0.3}\right\}^{0.2}}}{\left\{(0.64)^{0.1}(0.51)^{0.3}(0.75)^{0.3}(0.51)^{0.3}\right\}^{0.2}\left\{(0.36)^{0.1}(0.64)^{0.3}(0.36)^{0.3}(0.51)^{0.3}\right\}^{0.4}}\right\} . \\
& \left\{(1.36)^{0.1}(1.49)^{0.3}(1.25)^{0.3}(1.49)^{0.3}\right\}^{0.2}\left\{(1.64)^{0.1}(1.36)^{0.3}(1.64)^{0.3}(1.49)^{0.3}\right\}^{0.4} \\
& { }^{+}\left\{(0.84)^{0.1}(0.99)^{0.3}(0.75)^{0.3}(0.75)^{0.3}\right\}^{0.2}\left\{(0.75)^{0.1}(0.75)^{0.3}(0.64)^{0.3}(0.84)^{0.3}\right\}^{0.2} \\
& \left\{(0.64)^{0.1}(0.51)^{0.3}(0.75)^{0.3}(0.51)^{0.3}\right\}^{0.2}\left\{(0.36)^{0.1}(0.64)^{0.3}(0.36)^{0.3}(0.51)^{0.3}\right\}^{0.4}
\end{aligned}
$$

$0.4923,0.5743$

\subsection{Properties of PFSEOWG Operator}

3.1.1. Idempotency. Let $H_{i j}=\left(\mathfrak{a}_{i j}, \mathfrak{b}_{i j}\right)$ be a collection of PFSNs, where $(i=1,2, \ldots, n)$ and $(j=1,2, \ldots, m)$. If $H_{\mathfrak{r}(i) \mathfrak{z}(j)}=H_{11}$ are identical, then

$$
\operatorname{PFSEOWG}\left(H_{11}, H_{12}, \ldots, H_{n m}\right)=H \text {, }
$$

where $\mathcal{O}_{i}$ and $\mathscr{F}_{j}$ represent the weight vectors such that $\mathcal{O}_{i}>0, \sum_{i=1}^{n} \mathcal{O}_{i}=1$ and $\mathscr{J}_{j}>0, \sum_{j=1}^{n} \mathscr{J}_{j}=1$.

Proof. We know that PFSEOWG

$\operatorname{PFSEOWG}\left(H_{11}, H_{12}, \ldots, H_{n m}\right)$

$$
\begin{aligned}
& =\left\langle\frac{\sqrt{2 \prod_{j=1}^{m}\left(\prod_{i=1}^{n}\left(\mathfrak{a}_{\mathfrak{r}(i) \mathfrak{s}(j)}^{2}\right)^{\mathcal{G}_{i}}\right)^{\mathcal{F}_{j}}}}{\sqrt{\prod_{j=1}^{m}\left(\prod_{i=1}^{n}\left(2-\mathfrak{a}_{\mathfrak{r}(i) \mathfrak{F}(j)}^{2}\right)^{\mathcal{G}_{i}}\right)^{\mathcal{F}_{j}}+\prod_{j=1}^{m}\left(\prod_{i=1}^{n}\left(\mathfrak{a}_{\mathfrak{r}(i) \mathfrak{s}(j)}^{2}\right)^{\mathcal{G}_{i}}\right)^{\mathcal{F}_{j}}}},\right. \\
& \left.\cdot \frac{\sqrt{\prod_{j=1}^{m}\left(\prod_{i=1}^{n}\left(1+\mathfrak{b}_{\mathfrak{r}(i) \mathfrak{s}(j)}^{2}\right)^{\mathcal{G}_{i}}\right)^{\mathcal{F}_{j}}-\prod_{j=1}^{m}\left(\prod_{i=1}^{n}\left(1-\mathfrak{b}_{\mathfrak{r}(i) \mathfrak{s}(j)}^{2}\right)^{\mathcal{G}_{i}}\right)^{\mathcal{F}_{j}}}}{\prod_{j=1}^{m}\left(\prod_{i=1}^{n}\left(1+\mathfrak{b}_{\mathfrak{r}(i) \mathfrak{s}(j)}^{2}\right)^{\mathscr{G}_{i}}\right)^{\mathcal{G}_{j}}+\prod_{j=1}^{m}\left(\prod_{i=1}^{n}\left(1-\mathfrak{b}_{\mathfrak{r}(i) \mathfrak{s}(j)}^{2}\right)^{\mathcal{G}_{i}}\right)^{\mathcal{F}_{j}}}\right\rangle
\end{aligned}
$$


As $H_{\mathfrak{r}(i) \mathfrak{g}(j)}=H_{i j}$, so

$=\left\langle\frac{\sqrt{2\left(\left(\mathfrak{a}_{i j}^{2}\right)^{\sum_{i=1}^{n} \mathcal{G}_{i}}\right)^{\sum_{j=1}^{m} \mathcal{F}_{j}}}}{\sqrt{\left(\left(2-\mathfrak{a}_{i j}^{2}\right)^{\sum_{i=1}^{n} \mathcal{O}_{i}}\right)^{\sum_{j=1}^{m} \mathcal{F}_{j}}+\left(\left(\mathfrak{a}_{i j}^{2}\right)^{\sum_{i=1}^{n} \mathcal{O}_{i}}\right)^{\sum_{j=1}^{m} \mathcal{F}_{j}}}}\right.$,

$\left.\cdot \frac{\sqrt{\left(\left(1+\mathfrak{b}_{i j}^{2}\right)^{\sum_{i=1}^{n} \mathcal{O}_{i}}\right)^{\sum_{j=1}^{m} \mathcal{F}_{j}}-\left(\left(1-\mathfrak{b}_{i j}^{2}\right)^{\sum_{i=1}^{n} \mathcal{O}_{i}}\right)^{\sum_{j=1}^{m} \mathscr{F}_{j}}}}{\sqrt{\left(\left(1+\mathfrak{b}_{i j}^{2}\right)^{\sum_{i=1}^{n} \mathcal{O}_{i}}\right)^{\sum_{j=1}^{m} \mathcal{F}_{j}}+\left(\left(1-\mathfrak{b}_{i j}^{2}\right)^{\sum_{i=1}^{n} \mathcal{O}_{i}}\right)^{\sum_{j=1}^{m} \mathcal{F}_{j}}}}\right\rangle$

$=\left\langle\frac{\sqrt{2 \mathfrak{a}_{i j}^{2}}}{\sqrt{\left(2-\mathfrak{a}_{i j}^{2}\right)+\left(\mathfrak{a}_{i j}^{2}\right)}}, \frac{\sqrt{\left(1+\mathfrak{a}_{i j}^{2}\right)-\left(1-\mathfrak{a}_{i j}^{2}\right)}}{\sqrt{\left(1+\mathfrak{a}_{i j}^{2}\right)+\left(1-\mathfrak{a}_{i j}^{2}\right)}}\right\rangle=\mathfrak{a}_{i j}, \mathfrak{b}_{i j}=H$.
3.1.2. Boundedness. Let $H_{i j}=\left(\mathfrak{a}_{i j}, \mathfrak{b}_{i j}\right)$ be a collection of PFSNs, where $(i=1,2, \ldots, n)$ and $(j=1,2, \ldots, m)$, where $\mathcal{O}_{i}$ and $\mathscr{J}_{j}$ represent the weight vectors such that $\mathcal{O}_{i}>0$, $\sum_{i=1}^{n} \mathscr{O}_{i}=1 \quad$ and $\quad \mathscr{J}_{j}>0, \quad \sum_{j=1}^{n} \mathscr{J}_{j}=1 . \quad$ If $H_{\min }=\min \left(H_{\mathfrak{r}(i) \mathfrak{g}(j)}\right)$ and $H_{\max }=\max \left(H_{\mathfrak{r}(i) \mathfrak{z}(j)}\right)$, then $H_{\min } \leq$ PFSEOWG $\left(H_{11}, H_{12}, \ldots, H_{n m}\right) \leq H_{\text {max }}$.

Proof. Let $\left.\left.f(x)=\sqrt{2-x^{2} / x^{2}}, \quad x \in\right] 0,1\right]$, then $(\mathrm{d} / \mathrm{d} x)(f(x))=-2 / x^{3} \sqrt{x^{2} / 2-x^{2}}<0$. So, $f(x)$ is decreasing function on $] 0,1]$.

As $\quad \mathfrak{a}_{\min } \leq \mathfrak{a}_{\mathfrak{r}(i) \mathfrak{g}(j)} \leq \mathfrak{a}_{\max }, \quad \forall i, j, \quad$ so $\quad f\left(\mathfrak{a}_{\max }\right) \leq f$ $\left(\mathfrak{a}_{\mathfrak{r}(i) \mathfrak{\mathfrak { B }}(j)}\right) \leq f\left(\mathfrak{a}_{\min }\right) \quad$ and $\quad \sqrt{2-\mathfrak{a}_{\max }^{2} / \mathfrak{a}_{\max }^{2}} \leq$ $\sqrt{2-\mathfrak{a}_{\mathfrak{r}(i) \mathfrak{s}(j)}^{2} / \mathfrak{a}_{\mathfrak{r}(i) \mathfrak{s}(j)}^{2}} \leq \sqrt{2-\mathfrak{a}_{\min }^{2} / \mathfrak{a}_{\min }^{2}}$.

Let $\mathcal{O}_{i}$ and $\mathscr{F}_{j}$ represent the weight vectors such that $\mathcal{O}_{i}>0, \sum_{i=1}^{n} \mathcal{O}_{i}=1$ and $\mathscr{F}_{j}>0, \sum_{j=1}^{n} \mathscr{J}_{j}=1$, then we have

$\Leftrightarrow \sqrt{\prod_{j=1}^{m}\left(\prod_{i=1}^{n}\left(\frac{2-\mathfrak{a}_{\max }^{2}}{\mathfrak{a}_{\max }^{2}}\right)^{\mathcal{G}_{i}}\right)^{\mathcal{F}_{j}}} \leq \sqrt{\prod_{j=1}^{m}\left(\prod_{i=1}^{n}\left(\frac{2-\mathfrak{a}_{\mathfrak{r}(i) \mathfrak{s}(j)}^{2}}{\mathfrak{a}_{\mathfrak{r}(i) \mathfrak{s}(j)}^{2}}\right)^{\mathcal{G}_{i}}\right)^{\mathcal{F}_{j}}} \leq \sqrt{\prod_{j=1}^{m}\left(\prod_{i=1}^{n}\left(\frac{2-\mathfrak{a}_{\min }^{2}}{\mathfrak{a}_{\min }^{2}}\right)^{\mathcal{G}_{i}}\right)^{\mathcal{F}_{j}}}$

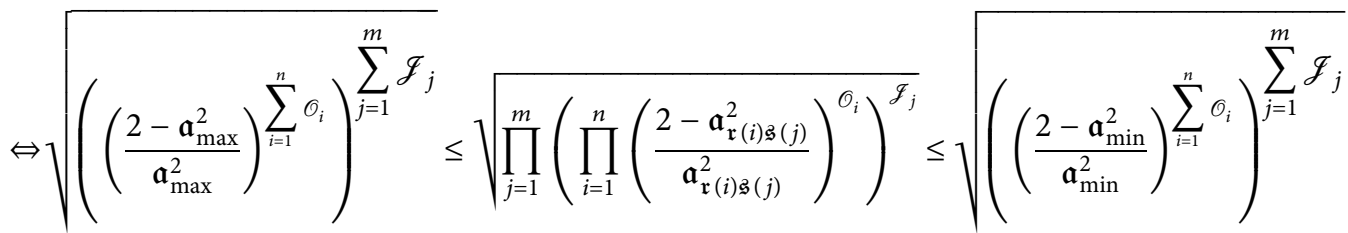

$\Leftrightarrow \sqrt{1+\left(\frac{2-\mathfrak{a}_{\max }^{2}}{\mathfrak{a}_{\max }^{2}}\right)} \leq \sqrt{1+\prod_{j=1}^{m}\left(\prod_{i=1}^{n}\left(\frac{2-\mathfrak{a}_{\mathfrak{r}(i) \mathfrak{s}(j)}^{2}}{\mathfrak{a}_{\mathfrak{r}(i) \mathfrak{s}(j)}^{2}}\right)^{\mathscr{G}_{i}}\right)^{\mathcal{F}_{j}}} \leq \sqrt{1+\left(\frac{2-\mathfrak{a}_{\min }^{2}}{\mathfrak{a}_{\min }^{2}}\right)}$

$\Leftrightarrow \sqrt{\frac{2}{\mathfrak{a}_{\max }^{2}}} \leq \sqrt{1+\prod_{j=1}^{m}\left(\prod_{i=1}^{n}\left(\frac{2-\mathfrak{a}_{\mathfrak{r}(i) \mathfrak{g}(j)}^{2}}{\mathfrak{a}_{\mathfrak{r}(i) \mathfrak{s}(j)}^{2}}\right)^{\mathscr{G}_{i}}\right)^{\mathcal{F}_{j}}} \leq \sqrt{\frac{2}{\mathfrak{a}_{\min }^{2}}}$

$\Leftrightarrow \sqrt{\frac{\mathfrak{a}_{\min }^{2}}{2}} \leq \frac{1}{\sqrt{1+\prod_{j=1}^{m}\left(\prod_{i=1}^{n}\left(2-\mathfrak{a}_{\mathfrak{r}(i) \mathfrak{s}(j)}^{2} / \mathfrak{a}_{\mathfrak{r}(i) \mathfrak{s}(j)}^{2}\right)^{\mathscr{G}_{i}}\right)^{\mathscr{F}_{j}}}} \leq \sqrt{\frac{\mathfrak{a}_{\max }^{2}}{2}}$

$\Leftrightarrow \mathfrak{a}_{\min } \leq \sqrt{\frac{2}{1+\prod_{j=1}^{m}\left(\prod_{i=1}^{n}\left(2-\mathfrak{a}_{\mathfrak{r}(i) \mathfrak{g}(j)}^{2} / \mathfrak{a}_{\mathfrak{r}(i) \mathfrak{s}(j)}^{2}\right)^{\mathscr{G}_{i}}\right)^{\mathcal{F}}}} \leq \mathfrak{a}_{\max }$

$\mathfrak{a}_{\min } \leq \frac{\sqrt{2 \prod_{j=1}^{m}\left(\prod_{i=1}^{n}\left(\mathfrak{a}_{\mathfrak{r}(i) \mathfrak{s}(j)}^{2}\right)^{\mathcal{G}_{i}}\right)^{\mathscr{F}_{j}}}}{\sqrt{\prod_{j=1}^{m}\left(\prod_{i=1}^{n}\left(2-\mathfrak{a}_{\mathfrak{r}(i) \mathfrak{s}(j)}^{2}\right)^{\mathscr{G}_{i}}\right)^{\mathcal{F}_{j}}+\prod_{j=1}^{m}\left(\prod_{i=1}^{n}\left(\mathfrak{a}_{\mathfrak{r}(i) \mathfrak{s}(j)}^{2}\right)^{\mathcal{G}_{i}}\right)^{\mathscr{F}_{j}}}} \leq \mathfrak{a}_{\max }$. 
Let $\left.\left.\quad g(y)=\sqrt{1-y^{2} / 1+y^{2}}, \quad y \in\right] 0,1\right], \quad$ then $(\mathrm{d} / \mathrm{d} y)(g(y))=-2 y /\left(1+y^{2}\right)^{2} \sqrt{1+y^{2} / 1-y^{2}}<0$, which shows that $g(y)$ is decreasing function on $] 0,1]$. So, $\mathfrak{b}_{\min } \leq \mathfrak{b}_{\mathfrak{r}(i) \mathfrak{s}(j)} \leq \mathfrak{b}_{\max }$.

Hence, $g\left(\mathfrak{b}_{\max }\right) \leq g\left(\mathfrak{b}_{\mathfrak{r}(i) \mathfrak{g}(j)}\right) \leq g\left(\mathfrak{b}_{\min }\right)$,

$$
\Rightarrow \sqrt{\frac{1-\mathfrak{b}_{\max }^{2}}{1+\mathfrak{b}_{\max }^{2}}} \leq \sqrt{\frac{1-\mathfrak{b}_{\mathfrak{r}(i) \mathfrak{s}(j)}^{2}}{1+\mathfrak{b}_{\mathfrak{r}(i) \mathfrak{s}(j) j}^{2}}} \leq \sqrt{\frac{1-\mathfrak{b}_{\min }^{2}}{1+\mathfrak{b}_{\min }^{2}}} .
$$

$$
\begin{aligned}
& \Leftrightarrow \sqrt{\prod_{j=1}^{m}\left(\prod_{i=1}^{n}\left(\frac{1-\mathfrak{b}_{\max }^{2}}{1+\mathfrak{b}_{\max }^{2}}\right)^{\mathcal{G}_{i}}\right)^{\mathcal{F}_{j}}} \leq \sqrt{\prod_{j=1}^{m}\left(\prod_{i=1}^{n}\left(\frac{1-\mathfrak{b}_{\mathfrak{r}(i) \mathfrak{g}(j)}^{2}}{1+\mathfrak{b}_{\mathfrak{r}(i) \mathfrak{B}(j)}^{2}}\right)^{\mathcal{G}_{i}}\right)^{\mathcal{F}_{j}}} \leq \sqrt{\prod_{j=1}^{m}\left(\prod_{i=1}^{n}\left(\frac{1-\mathfrak{b}_{\min }^{2}}{1+\mathfrak{b}_{\min }^{2}}\right)^{\mathcal{G}_{i}}\right)^{\mathcal{F}_{j}}},
\end{aligned}
$$

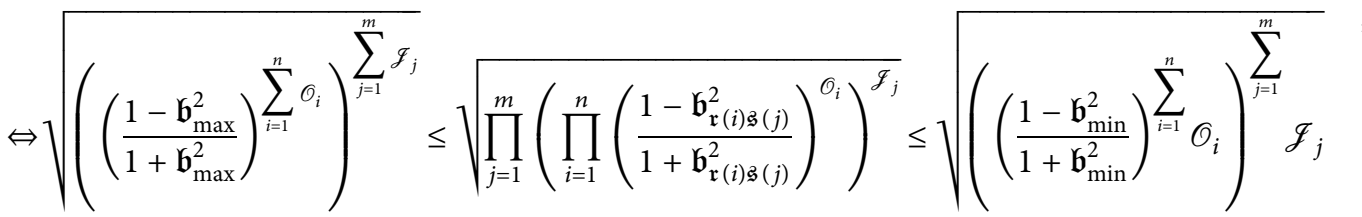

$$
\begin{aligned}
& \Leftrightarrow \sqrt{1+\left(\frac{1-\mathfrak{b}_{\max }^{2}}{1+\mathfrak{b}_{\max }^{2}}\right)} \leq \sqrt{1+\prod_{j=1}^{m}\left(\prod_{i=1}^{n}\left(\frac{1-\mathfrak{b}_{\mathfrak{r}(i) \mathfrak{s}(j)}^{2}}{1+\mathfrak{b}_{\mathfrak{r}(i) \mathfrak{B}(j)}^{2}}\right)^{\mathcal{G}_{i}}\right)^{\mathcal{F}_{j}}} \leq \sqrt{1+\left(\frac{1-\mathfrak{b}_{\min }^{2}}{1+\mathfrak{b}_{\min }^{2}}\right)}, \\
& \Leftrightarrow \sqrt{\frac{2}{1+\mathfrak{b}_{\max }^{2}}} \leq \sqrt{1+\prod_{j=1}^{m}\left(\prod_{i=1}^{n}\left(\frac{1-\mathfrak{b}_{\mathfrak{r}(i) \mathfrak{g}(j)}^{2}}{1+\mathfrak{b}_{\mathfrak{r}(i) \mathfrak{g}(j)}^{2}}\right)^{\mathcal{G}_{i}}\right)^{\mathcal{F}_{j}}} \leq \sqrt{\frac{2}{1+\mathfrak{b}_{\min }^{2}}} \\
& \Leftrightarrow \sqrt{\frac{1+\mathfrak{b}_{\min }^{2}}{2}} \leq \frac{1}{\sqrt{1+\prod_{j=1}^{m}\left(\prod_{i=1}^{n}\left(1-\mathfrak{b}_{\mathfrak{r}(i) \mathfrak{s}(j)}^{2} / 1+\mathfrak{b}_{\mathfrak{r}(i) \mathfrak{s}(j)}^{2}\right)^{\mathcal{G}_{i}}\right)^{\mathcal{F}_{j}}}} \leq \sqrt{\frac{1+\mathfrak{b}_{\max }^{2}}{2}} \\
& \Leftrightarrow \sqrt{1+\mathfrak{b}_{\min }^{2}} \leq \sqrt{\frac{2}{1+\prod_{j=1}^{m}\left(\prod_{i=1}^{n}\left(1-\mathfrak{b}_{\mathfrak{r}(i) \mathfrak{s}(j)}^{2} / 1+\mathfrak{b}_{\mathfrak{r}(i) \mathfrak{s}(j)}^{2}\right)^{\mathcal{G}_{i}}\right)^{\mathcal{F}_{j}}}} \leq \sqrt{1+\mathfrak{b}_{\max }^{2}}, \\
& \Leftrightarrow \sqrt{1+\mathfrak{b}_{\min }^{2}-1} \leq \sqrt{\frac{2}{1+\prod_{j=1}^{m}\left(\prod_{i=1}^{n}\left(1-\mathfrak{b}_{\mathfrak{r}(i) \mathfrak{s}(j)}^{2} / 1+\mathfrak{b}_{\mathfrak{r}(i) \mathfrak{s}(j)}^{2}\right)^{\mathscr{G}_{i}}\right)^{\mathcal{F}}}-1} \leq \sqrt{1+\mathfrak{b}_{\max }^{2}-1}, \\
& \Leftrightarrow \sqrt{\mathfrak{b}_{\min }^{2}} \leq \sqrt{\frac{2}{1+\prod_{j=1}^{m}\left(\prod_{i=1}^{n}\left(1-\mathfrak{b}_{\mathfrak{r}(i) \mathfrak{g}(j)}^{2} / 1+\mathfrak{b}_{\mathfrak{r}(i) \mathfrak{s}(j)}^{2}\right)^{G_{i}}\right)^{\mathcal{F}_{j}}}-1} \leq \sqrt{\mathfrak{b}_{\max }^{2}}, \\
& \Leftrightarrow \mathfrak{b}_{\min } \leq \sqrt{\frac{2}{1+\prod_{j=1}^{m}\left(\prod_{i=1}^{n}\left(1-\mathfrak{b}_{\mathfrak{r}(i) \mathfrak{g}(j)}^{2} / 1+\mathfrak{b}_{\mathfrak{r}(i) \mathfrak{s}(j)}^{2}\right)^{\mathcal{G}_{i}}\right)^{\mathcal{F}_{j}}}-1} \leq \mathfrak{b}_{\max }, \\
& \mathfrak{b}_{\min } \leq \frac{\sqrt{\prod_{j=1}^{m}\left(\prod_{i=1}^{n}\left(1+\mathfrak{b}_{\mathfrak{r}(i) \mathfrak{s}(j)}^{2}\right)^{\mathscr{G}_{i}}\right)^{\mathcal{F}_{j}}-\prod_{j=1}^{m}\left(\prod_{i=1}^{n}\left(1-\mathfrak{b}_{\mathfrak{r}(i) \mathfrak{s}(j)}^{2}\right)^{\mathscr{G}_{i}}\right)^{\mathcal{F}_{j}}}}{\sqrt{\prod_{j=1}^{m}\left(\prod_{i=1}^{n}\left(1+\mathfrak{b}_{\mathfrak{r}(i) \mathfrak{s}(j)}^{2}\right)^{\mathcal{G}_{i}}\right)^{\mathcal{F}_{j}}+\prod_{j=1}^{m}\left(\prod_{i=1}^{n}\left(1-\mathfrak{b}_{\mathfrak{r}(i) \mathfrak{s}(j)}^{2}\right)^{\mathcal{G}_{i}}\right)^{\mathcal{F}_{j}}}} \leq \mathfrak{b}_{\max } .
\end{aligned}
$$


Let $\operatorname{PFSEOWG}\left(H_{11}, H_{12}, \ldots, H_{\mathrm{nm}}\right)=\mathscr{H}$.

Then, inequalities (21) and (23) can be written as $\leq \mathfrak{a} \leq \mathfrak{a}_{\max }$ and $\mathfrak{b}_{\min } \leq \mathfrak{b} \leq \mathfrak{b}_{\max }$

Thus, $S(H)=\mathfrak{a}^{2}-\mathfrak{b}^{2} \leq \mathfrak{a}_{\max }^{2}-\mathfrak{b}_{\min }^{2}=S\left(H_{\max }\right)$ and $S$ $(H)=\mathfrak{a}^{2}-\mathfrak{b}^{2} \geq \mathfrak{a}_{\min }^{2}-\mathfrak{b}_{\max }^{2}=S\left(H_{\min }\right)$.

If $S(H)<S\left(H_{\max }\right)$ and $S(H)>S\left(H_{\min }\right)$, then we have

$H_{\text {min }}<\operatorname{PFSEOW}\left(H_{11}, H_{12}, \ldots, H_{\mathrm{nm}}\right)<H_{\max }$.

If $S(H)=S\left(H_{\max }\right)$, then we have $\mathfrak{a}^{2}=\mathfrak{a}_{\max }^{2}$ and $\mathfrak{b}^{2}=\mathfrak{b}_{\max }^{2}$. Thus, $A(H)=\mathfrak{a}^{2}+\mathfrak{b}^{2}=\mathfrak{a}_{\max }^{2}+\mathfrak{b}_{\max }^{2}=A\left(H_{\max }\right)$. Therefore,

$$
\operatorname{PFSEOWG}\left(H_{11}, H_{12}, \ldots, H_{\mathrm{nm}}\right)=H_{\text {max }} .
$$

If $S(H)=S\left(H_{\min }\right)$. Then we have $\mathfrak{a}^{2}-\mathfrak{b}^{2}=\mathfrak{a}_{\min }^{2}-\mathfrak{b}_{\min }^{2}$, $\mathfrak{a}^{2}=\mathfrak{a}_{\min }^{2}$, and $\mathfrak{b}^{2}=\mathfrak{b}_{\min }^{2}$.

Thus, A $(H)=\mathfrak{a}^{2}+\mathfrak{b}^{2}=\mathfrak{a}_{\text {min }}^{2}+\mathfrak{b}_{\text {min }}^{2}=A \quad\left(H_{\min }\right)$. Therefore,

$$
\begin{aligned}
& \operatorname{PFSEOWG}\left(H_{11}, H_{12}, \ldots, H_{\mathrm{nm}}\right)=H_{\text {min }} . \\
& \begin{array}{c}
\text { Hence, } \\
\left.H_{\mathrm{nm}}\right) \leq H_{\max } .
\end{array}
\end{aligned}
$$

3.1.3. Homogeneity. Prove that PFSEOWG $\left(H_{11}, H_{12}, \ldots\right.$, $\left.H_{\mathrm{nm}}\right)=\partial$ PFSEOWG $\left(H_{11}, H_{12}, \ldots, H_{\mathrm{nm}}\right)$ for any positive real number $\partial$.

Proof. Let $\mathrm{H}_{\mathrm{ij}}$ be a PFSN and $\partial>0 . T$, then we know that $\partial H_{i j}=\left\langle\frac{\sqrt{2\left(\mathfrak{a}^{2}\right)^{\partial}}}{\sqrt{\left(2-\mathfrak{a}^{2}\right)^{\partial}+\left(\mathfrak{a}^{2}\right)^{\partial}}}, \frac{\sqrt{\left(1+\mathfrak{b}^{2}\right)^{\partial}-\left(1-\mathfrak{b}^{2}\right)^{\partial}}}{\sqrt{\left(1+\mathfrak{b}^{2}\right)^{\partial}+\left(1-\mathfrak{b}^{2}\right)^{\partial}}}\right\rangle$

So,

$$
\begin{aligned}
& \text { PFSEOWG }=\left(H_{11}, H_{12}, \ldots, H_{n m}\right) \\
& =\left\langle\frac{\sqrt{2 \prod_{j=1}^{m}\left(\prod_{i=1}^{n}\left(\mathfrak{a}_{\mathfrak{r}(i) \mathfrak{z}(j)}^{2}\right)^{\mathcal{G}_{i}}\right)^{\mathcal{F}_{j}}}}{\sqrt{\prod_{j=1}^{m}\left(\prod_{i=1}^{n}\left(2-\mathfrak{a}_{\mathfrak{r}(i) \mathfrak{g}(j)}^{2}\right)^{\mathcal{G}_{i}}\right)^{\mathcal{F}_{j}}+\prod_{j=1}^{m}\left(\prod_{i=1}^{n}\left(\mathfrak{a}_{\mathfrak{r}(i) \mathfrak{g}(j)}^{2}\right)^{\mathcal{G}_{i}}\right)^{\mathcal{F}_{j}}}},\right. \\
& \left.\cdot \frac{\sqrt{\prod_{j=1}^{m}\left(\prod_{i=1}^{n}\left(1+\mathfrak{b}_{\mathfrak{r}(i) \mathfrak{z}(j)}^{2}\right)^{\mathcal{G}_{i}}\right)^{\mathcal{F}_{j}}-\prod_{j=1}^{m}\left(\prod_{i=1}^{n}\left(1-\mathfrak{b}_{\mathfrak{r}(i) \mathfrak{g}(j)}^{2}\right)^{\mathcal{G}_{i}}\right)^{\mathcal{F}_{j}}}}{\sqrt{\prod_{j=1}^{m}\left(\prod_{i=1}^{n}\left(1+\mathfrak{b}_{\mathfrak{r}(i) \mathfrak{g}(j)}^{2}\right)^{\mathcal{G}_{i}}\right)^{\mathcal{F}_{j}}+\prod_{j=1}^{m}\left(\prod_{i=1}^{n}\left(1-\mathfrak{b}_{\mathfrak{r}(i) \mathfrak{z}(j)}^{2}\right)^{\mathcal{G}_{i}}\right)^{\mathcal{F}_{j}}}}\right\rangle \\
& \left\langle\frac{\sqrt{\left(2 \prod_{j=1}^{m}\left(\prod_{i=1}^{n}\left(\mathfrak{a}_{\mathfrak{r}(i) \mathfrak{s}(j)}^{2}\right)^{\mathcal{O}_{i}}\right)^{\mathcal{F}_{j}}\right)^{\partial}}}{\sqrt{\left(\prod_{j=1}^{m}\left(\prod_{i=1}^{n}\left(2-\mathfrak{a}_{\mathfrak{r}(i) \mathfrak{s}(j)}^{2}\right)^{\mathcal{G}_{i}}\right)^{\mathcal{F}_{j}}\right)^{\partial}}+\left(\prod_{j=1}^{m}\left(\prod_{i=1}^{n}\left(\mathfrak{a}_{\mathfrak{r}(i) \mathfrak{I}(j)}^{2}\right)^{\mathcal{G}_{i}}\right)^{\mathcal{F}_{j}}\right)^{\partial}},\right.
\end{aligned}
$$

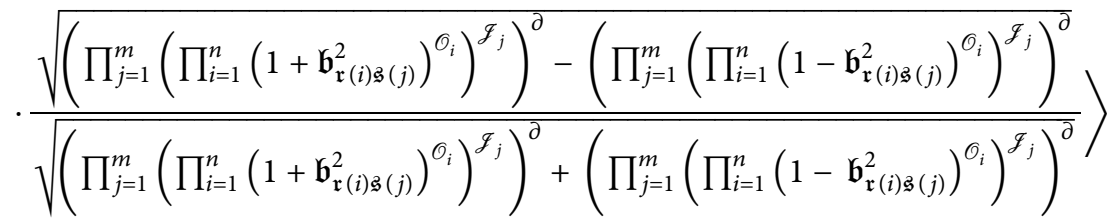

$$
\begin{aligned}
& =\partial \operatorname{PFSEOWG}\left(H_{11}, H_{12}, \ldots, H_{n m}\right)
\end{aligned}
$$

\section{Multiattribute Group Decision- Making Approach}

This section develops a DM technique to resolve the MAGDM complication using our proposed PFSEOWG operator and a numerical example.

4.1. Proposed Approach. Let $S=\left\{S^{1}, S^{2}, S^{3}, \ldots, S^{s}\right\}$ be the set of $s$ alternatives, $W=\left\{W_{1}, W_{2}, W_{3}, \ldots, W_{r}\right\}$ be the set of $r$ experts (decision makers), and $\mathbb{N}=\left\{t_{1}, t_{2}, t_{3}, \ldots, t_{m}\right\}$ be the set of $m$ attributes. Let weighted vector of experts $W(i=1,2,3, \ldots, r)$ be $O=\left(\mathcal{O}_{1}, \mathcal{O}_{2}, \mathcal{O}_{3}, \ldots, \mathcal{O}_{n}\right)^{T}$ such that $\mathcal{O}_{i}>0, \quad \sum_{i=1}^{n} \mathcal{O}_{i}=1$. Let weight vector of attributes $t_{i}(i=$ $1,2,3, \ldots, m)$ be $\mathscr{J}=\left(\mathscr{J}_{1}, \mathscr{J}_{2}, \mathscr{J}_{3}, \ldots, \mathscr{J}_{n}\right)^{T}$ such that $\mathscr{J}_{j}>0, \sum_{j=1}^{n} \mathscr{J}_{j}=1$. Team of experts $O_{i}(i=1,2,3, \ldots, r)$ consider the alternatives $S^{i}(i=1,2,3, \ldots, s)$ for attributes in the form of PFSNs such as $F=\left(\mathscr{H}_{i j}\right)_{n * m}=\left(\mathfrak{a}_{i j}, \mathfrak{b}_{i j}\right)_{n * m}$, 
TABLE 1: PFS decision matrix for $S^{1}$.

\begin{tabular}{ccccc}
\hline & $t_{1}$ & $t_{2}$ & $t_{3}$ & $t_{4}$ \\
\hline$O^{1}$ & $(0.8,0.5)$ & $(0.7,0.5)$ & $(0.6,0.4)$ & $(0.7,0.4)$ \\
$O^{2}$ & $(0.6,0.5)$ & $(0.9,0.1)$ & $(0.7,0.3)$ & $(0.4,0.5)$ \\
$O^{3}$ & $(0.8,0.4)$ & $(0.7,0.5)$ & $(0.6,0.4)$ & $(0.3,0.5)$ \\
$O^{4}$ & $(0.7,0.3)$ & $(0.6,0.5)$ & $(0.4,0.5)$ & $(0.5,0.7)$ \\
\hline
\end{tabular}

TABLE 2: PFS decision matrix for $S^{2}$.

\begin{tabular}{ccccc}
\hline & $t_{1}$ & $t_{2}$ & $t_{3}$ & $t_{4}$ \\
\hline$O^{1}$ & $(0.7,0.5)$ & $(0.8,0.5)$ & $(0.6,0.4)$ & $(0.8,0.4)$ \\
$O^{2}$ & $(0.6,0.3)$ & $(0.9,0.2)$ & $(0.8,0.3)$ & $(0.7,0.5)$ \\
$O^{3}$ & $(0.5,0.4)$ & $(0.6,0.5)$ & $(0.6,0.3)$ & $(0.3,0.6)$ \\
$O^{4}$ & $(0.7,0.4)$ & $(0.6,0.4)$ & $(0.7,0.5)$ & $(0.5,0.7)$ \\
\hline
\end{tabular}

TABLe 3: PFS decision matrix for $S^{3}$.

\begin{tabular}{ccccc}
\hline & $t_{1}$ & $t_{2}$ & $t_{3}$ & $t_{4}$ \\
\hline$O^{1}$ & $(0.7,0.5)$ & $(0.7,0.4)$ & $(0.6,0.4)$ & $(0.8,0.4)$ \\
$O^{2}$ & $(0.6,0.6)$ & $(0.9,0.1)$ & $(0.6,0.3)$ & $(0.4,0.5)$ \\
$O^{3}$ & $(0.8,0.3)$ & $(0.7,0.2)$ & $(0.6,0.5)$ & $(0.4,0.5)$ \\
$O^{4}$ & $(0.7,0.6)$ & $(0.3,0.5)$ & $(0.4,0.5)$ & $(0.5,0.6)$ \\
\hline
\end{tabular}

TABLE 4: PFS decision matrix for $S^{4}$.

\begin{tabular}{ccccc}
\hline & $t_{1}$ & $t_{2}$ & $t_{3}$ & $t_{4}$ \\
\hline$O^{1}$ & $(0.8,0.5)$ & $(0.7,0.5)$ & $(0.7,0.4)$ & $(0.6,0.4)$ \\
$O^{2}$ & $(0.6,0.4)$ & $(0.8,0.1)$ & $(0.7,0.3)$ & $(0.4,0.7)$ \\
$O^{3}$ & $(0.7,0.4)$ & $(0.7,0.5)$ & $(0.6,0.4)$ & $(0.3,0.5)$ \\
$O^{4}$ & $(0.6,0.3)$ & $(0.6,0.3)$ & $(0.8,0.5)$ & $(0.5,0.6)$ \\
\hline
\end{tabular}

TABLe 5: PFS decision matrix for $S^{5}$.

\begin{tabular}{ccccc}
\hline & $t_{1}$ & $t_{2}$ & $t_{3}$ & $t_{4}$ \\
\hline$O^{1}$ & $(0.6,0.5)$ & $(0.6,0.5)$ & $(0.6,0.4)$ & $(0.5,0.4)$ \\
$O^{2}$ & $(0.6,0.4)$ & $(0.8,0.1)$ & $(0.8,0.3)$ & $(0.7,0.5)$ \\
$O^{3}$ & $(0.6,0.4)$ & $(0.7,0.3)$ & $(0.6,0.4)$ & $(0.6,0.5)$ \\
$O^{4}$ & $(0.7,0.4)$ & $(0.7,0.5)$ & $(0.4,0.5)$ & $(0.5,0.8)$ \\
\hline
\end{tabular}

where $0 \leq \mathfrak{a}_{i j}, \mathfrak{b}_{i j} \leq 1$ and $0 \leq \mathfrak{a}_{i j}^{2}, \mathfrak{b}_{i j}^{2} \leq 1 \forall i, j$ are given in Tables 1-5.

We will apply the proposed PFSEOWG operator to solve the MAGDM problem, which has the following steps:

Step-1: obtain decision matrices $F=\left(H_{i j}\right)_{n * m}$ for alternatives relative to attributes in the form of PFSNs.

Step-2: find the associated ordered position matrix by using the score function.

Step-3: by using the normalization formula, normalize the decision matrix to convert the rating value of cost type parameters into benefit type parameters.

$$
M_{i j}=\left\{\begin{array}{l}
\mathscr{H}_{i j}^{c}=\left(b_{i j}, \alpha_{i j}\right) \text { cost type parameter } \\
F=\left(H_{i j}\right)_{n * m} \text { benefit type parameter. }
\end{array}\right.
$$

Step-4: use the developed PFSEOWG operator to aggregate the PFSNs $H_{i j}$ for each alternative $S=\left\{S^{1}, S^{2}, S^{3}, \ldots, S^{s}\right\}$ into the decision matrix $H_{i j}$.

Step-5: calculate the score values of $H$ for all alternatives by using equation (7).

Step-6: select the alternative which has the maximum score value and examine the ranking.

4.2. Numerical Example. Suppose a rider has to choose a bike, and he has five alternatives such as $S^{1}$, Jawa Perak $t$; $S^{2}$, KTM 390 Duke; $S^{3}$, Honda CBR 250R; $S^{4}$, Bajaj Avenger Cruise 220; and $S^{5}$, Royal Enfield Himalayan. There are four considered attributes, according to which rider must have to take decision such as $t_{1}$, size; $t_{2}$, compatibility; $t_{3}$, maintenance; and $t_{4}$, aesthetics, with the weight vector $\mathscr{J}=(0.2,0.2,0.2,0.4)^{T}$. Here, $t_{1}, t_{3}$ are cost type parameters and $t_{2}, t_{4}$ are benefit type parameters. Businessmen hire a team of four experts $O_{r}(r=1,2,3,4)$ for decision-making with the weight vector $\mathcal{O}=(0.1,0.3,0.3,0.3)^{T}$.

Step-1: according to the expert's opinion, Pythagorean fuzzy soft decision matrices for all alternatives are given in Tables 1-5.

Step-2: according to experts' opinions, the ordered Pythagorean fuzzy soft decision matrix for finding all alternatives is shown in Tables 6-10.

Step-3: because $t_{1}$ and $t_{3}$ represent the cost type parameters. Therefore, the normalized ordered Pythagorean fuzzy soft decision matrix is obtained using the normalized formula in Tables 11-15.

Step-4: the proposed PFSEOWG operator was applied to the acquired data, and then we obtain the opinions of decision makers on each alternative in the form of PFSNs such as $H_{1}=0.3203,0.8382, H_{2}=0.4912$, $0.6112, H_{3}=0.4675,0.5801, H_{4}=0.5421,0.6512$, and $H_{5}=0.4821,0.5994$.

Step-5: use the score formula $\mathfrak{H}=\alpha_{i j}^{2}-b_{i j}^{2}$ to calculate the score values for all alternatives.

$$
\begin{aligned}
& \mathfrak{N}\left(H_{1}\right)=-0.5999, \\
& \mathfrak{H}\left(H_{2}\right)=-0.1322, \\
& \mathfrak{H}\left(H_{3}\right)=-0.1179, \\
& \mathfrak{S}\left(H_{4}\right)=-0.1301, \\
& \mathfrak{S}\left(H_{5}\right)=-0.1068 .
\end{aligned}
$$

Step-6: after calculation, we get the ranking of alternatives $\mathfrak{H}\left(\mathscr{H}_{5}\right)>\mathfrak{H}\left(\mathscr{H}_{3}\right)>\mathfrak{H}\left(\mathscr{H}_{4}\right)>\mathfrak{H}\left(\mathscr{H}_{2}\right)>\mathfrak{H}\left(\mathscr{H}_{1}\right)$. So, $S^{5}>S^{3}>S^{4}>S^{2}>S^{1}$.

Hence, the best alternative is $S^{5}$.

\section{Comparative Studies}

It is compared with some existing PFS and PFSS to demonstrate the efficiency of the anticipated model. 
TABLE 6: Ordered PFS decision matrix for $S^{1}$.

\begin{tabular}{ccccc}
\hline & $t_{1}$ & $t_{2}$ & $t_{3}$ & $t_{4}$ \\
\hline$O^{1}$ & $(0.8,0.5)$ & $(0.7,0.5)$ & $(0.6,0.4)$ & $(0.7,0.4)$ \\
$O^{2}$ & $(0.6,0.5)$ & $(0.9,0.1)$ & $(0.7,0.3)$ & $(0.4,0.5)$ \\
$O^{3}$ & $(0.8,0.4)$ & $(0.7,0.5)$ & $(0.6,0.4)$ & $(0.3,0.5)$ \\
$O^{4}$ & $(0.7,0.3)$ & $(0.6,0.5)$ & $(0.4,0.5)$ & $(0.5,0.7)$ \\
\hline
\end{tabular}

TABLE 7: Ordered PFS decision matrix for $S^{2}$.

\begin{tabular}{ccccc}
\hline & $t_{1}$ & $t_{2}$ & $t_{3}$ & $t_{4}$ \\
\hline$O^{1}$ & $(0.7,0.5)$ & $(0.8,0.5)$ & $(0.6,0.4)$ & $(0.8,0.4)$ \\
$O^{2}$ & $(0.6,0.3)$ & $(0.9,0.2)$ & $(0.8,0.3)$ & $(0.7,0.5)$ \\
$O^{3}$ & $(0.5,0.4)$ & $(0.6,0.5)$ & $(0.6,0.3)$ & $(0.3,0.6)$ \\
$O^{4}$ & $(0.7,0.4)$ & $(0.6,0.4)$ & $(0.7,0.5)$ & $(0.5,0.7)$ \\
\hline
\end{tabular}

TABLE 8: Ordered PFS decision matrix for $S^{3}$.

\begin{tabular}{ccccc}
\hline & $t_{1}$ & $t_{2}$ & $t_{3}$ & $t_{4}$ \\
\hline$O^{1}$ & $(0.7,0.5)$ & $(0.7,0.4)$ & $(0.6,0.4)$ & $(0.8,0.4)$ \\
$O^{2}$ & $(0.6,0.6)$ & $(0.9,0.1)$ & $(0.6,0.3)$ & $(0.4,0.5)$ \\
$O^{3}$ & $(0.8,0.3)$ & $(0.7,0.2)$ & $(0.6,0.5)$ & $(0.4,0.5)$ \\
$O^{4}$ & $(0.7,0.6)$ & $(0.3,0.5)$ & $(0.4,0.5)$ & $(0.5,0.6)$ \\
\hline
\end{tabular}

TABle 9: Ordered PFS decision matrix for $S^{4}$.

\begin{tabular}{ccccc}
\hline & $t_{1}$ & $t_{2}$ & $t_{3}$ & $t_{4}$ \\
\hline$O^{1}$ & $(0.8,0.5)$ & $(0.7,0.5)$ & $(0.7,0.4)$ & $(0.6,0.4)$ \\
$O^{2}$ & $(0.6,0.4)$ & $(0.8,0.1)$ & $(0.7,0.3)$ & $(0.4,0.7)$ \\
$O^{3}$ & $(0.7,0.4)$ & $(0.7,0.5)$ & $(0.6,0.4)$ & $(0.3,0.5)$ \\
$O^{4}$ & $(0.6,0.3)$ & $(0.6,0.3)$ & $(0.8,0.5)$ & $(0.5,0.6)$ \\
\hline
\end{tabular}

Table 10: Ordered PFS decision matrix for $S^{5}$.

\begin{tabular}{ccccc}
\hline & $t_{1}$ & $t_{2}$ & $t_{3}$ & $t_{4}$ \\
\hline$O^{1}$ & $(0.6,0.5)$ & $(0.6,0.5)$ & $(0.6,0.4)$ & $(0.5,0.4)$ \\
$O^{2}$ & $(0.6,0.4)$ & $(0.8,0.1)$ & $(0.8,0.3)$ & $(0.7,0.5)$ \\
$O^{3}$ & $(0.6,0.4)$ & $(0.7,0.3)$ & $(0.6,0.4)$ & $(0.6,0.5)$ \\
$O^{4}$ & $(0.7,0.4)$ & $(0.7,0.5)$ & $(0.4,0.5)$ & $(0.5,0.8)$ \\
\hline
\end{tabular}

TABLE 11: Normalized ordered PFS decision matrix for $S^{1}$.

\begin{tabular}{ccccc}
\hline & $t_{1}$ & $t_{2}$ & $t_{3}$ & $t_{4}$ \\
\hline$O^{1}$ & $(0.5,0.8)$ & $(0.7,0.5)$ & $(0.4,0.6)$ & $(0.7,0.4)$ \\
$O^{2}$ & $(0.5,0.6)$ & $(0.9,0.1)$ & $(0.3,0.7)$ & $(0.4,0.5)$ \\
$O^{3}$ & $(0.4,0.8)$ & $(0.7,0.5)$ & $(0.4,0.6)$ & $(0.3,0.5)$ \\
$O^{4}$ & $(0.3,0.7)$ & $(0.6,0.5)$ & $(0.5,0.4)$ & $(0.5,0.7)$ \\
\hline
\end{tabular}

5.1. Advantages of the Proposed Method. The predictable procedure is practical and reasonable; we have an innovative approach under the PFSS setting over the PFSEOWG operator. Our expected model is more talented than conventional structures and can carry the most
TABLE 12: Normalized ordered PFS decision matrix for $S^{2}$.

\begin{tabular}{ccccc}
\hline & $t_{1}$ & $t_{2}$ & $t_{3}$ & $t_{4}$ \\
\hline$O^{1}$ & $(0.5,0.7)$ & $(0.8,0.5)$ & $(0.4,0.6)$ & $(0.8,0.4)$ \\
$O^{2}$ & $(0.3,0.6)$ & $(0.9,0.2)$ & $(0.3,0.8)$ & $(0.7,0.5)$ \\
$O^{3}$ & $(0.4,0.5)$ & $(0.6,0.5)$ & $(0.3,0.6)$ & $(0.3,0.6)$ \\
$O^{4}$ & $(0.4,0.7)$ & $(0.6,0.4)$ & $(0.5,0.7)$ & $(0.5,0.7)$ \\
\hline
\end{tabular}

TABLE 13: Normalized ordered PFS decision matrix for $S^{3}$.

\begin{tabular}{ccccc}
\hline & $t_{1}$ & $t_{2}$ & $t_{3}$ & $t_{4}$ \\
\hline$O^{1}$ & $(0.5,0.7)$ & $(0.7,0.4)$ & $(0.4,0.6)$ & $(0.8,0.4)$ \\
$O^{2}$ & $(0.6,0.6)$ & $(0.9,0.1)$ & $(0.3,0.6)$ & $(0.4,0.5)$ \\
$O^{3}$ & $(0.3,0.8)$ & $(0.7,0.2)$ & $(0.5,0.6)$ & $(0.4,0.5)$ \\
$O^{4}$ & $(0.6,0.7)$ & $(0.3,0.5)$ & $(0.5,0.4)$ & $(0.5,0.6)$ \\
\hline
\end{tabular}

TABLE 14: Normalized ordered PFS decision matrix for $S^{4}$.

\begin{tabular}{ccccc}
\hline & $t_{1}$ & $t_{2}$ & $t_{3}$ & $t_{4}$ \\
\hline$O^{1}$ & $(0.5,0.8)$ & $(0.7,0.5)$ & $(0.4,0.7)$ & $(0.6,0.4)$ \\
$O^{2}$ & $(0.4,0.6)$ & $(0.8,0.1)$ & $(0.3,0.7)$ & $(0.4,0.7)$ \\
$O^{3}$ & $(0.4,0.7)$ & $(0.7,0.5)$ & $(0.4,0.6)$ & $(0.3,0.5)$ \\
$O^{4}$ & $(0.3,0.6)$ & $(0.6,0.3)$ & $(0.5,0.8)$ & $(0.5,0.6)$ \\
\hline
\end{tabular}

TABLE 15: Normalized PFS ordered decision matrix for $S^{5}$.

\begin{tabular}{ccccc}
\hline & $t_{1}$ & $t_{2}$ & $t_{3}$ & $t_{4}$ \\
\hline$O^{1}$ & $(0.5,0.6)$ & $(0.6,0.5)$ & $(0.4,0.6)$ & $(0.5,0.4)$ \\
$O^{2}$ & $(0.4,0.6)$ & $(0.8,0.1)$ & $(0.3,0.8)$ & $(0.7,0.5)$ \\
$O^{3}$ & $(0.4,0.6)$ & $(0.7,0.3)$ & $(0.4,0.6)$ & $(0.6,0.5)$ \\
$O^{4}$ & $(0.4,0.7)$ & $(0.7,0.5)$ & $(0.5,0.4)$ & $(0.5,0.8)$ \\
\hline
\end{tabular}

progressive values in MAGDM difficulties. The merger model is multipurpose and familiar to accommodate potential variations, involvement, and efficiency. Different models have specific ranking measures, so there are incidental alterations among the grades of the projected technique to be reasonable based on their deliberations. Based on this scientific study and estimation, we now accomplish that the consequences attained from standing approaches are impulsively associated with hybrid structures. In addition, due to some prosperous environments, numerous hybrid assemblies of FS and IFS have to convert infrequently for PFSS. Thus, it is a modest method to combine inadequate and inexact information in the DM technique. Therefore, our planned technique will be more competent, more significant, improved, and healthier than the numerous other mix structures of FS. Table 16 shows the characteristic analysis of the anticipated technique and some current models.

5.2. Comparative Analysis. To confirm the effectiveness of the proposed method, we compared the results obtained with some existing techniques in the PFSS setting. All results are summarized in Table 17. In this work, a new aggregation 
TABLE 16: Characteristic analysis of different approaches.

\begin{tabular}{lcccc}
\hline & $\begin{array}{c}\text { Fuzzy } \\
\text { information }\end{array}$ & $\begin{array}{c}\text { Aggregated parameters } \\
\text { information }\end{array}$ & $\begin{array}{c}\text { Aggregated parameters information considering the Einstein } \\
\text { norm }\end{array}$ \\
\hline IFWA [3] & $\checkmark$ & $\times$ & $\times$ \\
IFEWG [4] & $\checkmark$ & $\times$ & $\checkmark$ & $\times$ \\
PFWG [8] & $\checkmark$ & $\times$ & $\checkmark$ & \\
PFEWG [31] & $\checkmark$ & $\times$ & $\checkmark$ & $\times$ \\
PFEHA [32] & $\checkmark$ & $\times$ & $\checkmark$ \\
IFSWG [33] & $\checkmark$ & $\checkmark$ & $\checkmark$ \\
PFSWG [25] & $\checkmark$ & $\checkmark$ & $\checkmark$ \\
Proposed & $\checkmark$ & $\checkmark$ & \\
operator & & & \\
\hline
\end{tabular}

TABLE 17: Comparison of the planned operator with some prevailing operators.

\begin{tabular}{|c|c|c|c|c|c|c|}
\hline Approach & $S^{1}$ & $S^{2}$ & $S^{3}$ & $S^{4}$ & $S^{5}$ & Alternatives ranking \\
\hline Proposed operator & -0.5999 & -0.1322 & -0.1179 & -0.1301 & -0.1068 & $S^{5}>S^{3}>S^{4}>S^{2}>S^{1}$ \\
\hline PFSWA [25] & 0.0293 & 0.0369 & 0.0783 & -0.0938 & 0.0858 & $S^{5}>S^{3}>S^{1}>S^{4}>S^{2}$ \\
\hline PFSWG [25] & -0.3306 & -0.1383 & -0.1092 & -0.1661 & -0.5957 & $S^{5}>S^{3}>S^{1}>S^{4}>S^{2}$ \\
\hline PFSEWA [32] & 0.0039 & -0.0376 & 0.0433 & -0.0179 & 0.0644 & $S^{5}>S^{3}>S^{1}>S^{4}>S^{2}$ \\
\hline PFSEWG [31] & -0.4975 & -0.1204 & -0.1775 & -0.0778 & -0.1211 & $S^{5}>S^{2}>S^{4}>S^{3}>S^{1}$ \\
\hline
\end{tabular}

operator, the PFSEOWG operator, is recommended to fuse indicative information and then use the score function to evaluate the classification of alternatives. Also, if only one parameter is assumed instead of one, then the PFSS theory becomes easier in PFS. Therefore, PFSS theory is a general form of PFS theory. Consequently, it is true that based on the above facts, the operator proposed in this work is more robust, more reliable, and more successful.

It is also a suitable tool for outrageous wrong and erroneous information in the DM procedure. Compared with contemporary approaches, the benefit of the intended technique and associated dealings is to avoid implications based on detested reasons. So, it is an appropriate tool for merging erroneous and imprecise data in the DM.

\section{Conclusion}

The PFSS is more operative than IFSS and PFS because they resolve incomplete and uncertain information using $\mathrm{MG}$ and NMG. AO is a mathematical tool that actively reduces fuzzy numbers to a single fuzzy number. This paper develops the novel AO for PFSS, such as the PFSEOWG operator. In addition, some basic properties are also proposed, such as the idempotency, homogeneity, and boundedness of the developed PFSEOWG operator. Moreover, a DM technique has been offered to resolve the complications of MAGDM using the projected operator. A comprehensive mathematical illustration is prearranged to choose the best bicycle for the rider to confirm the effectiveness of the recognized technique. A comparative analysis with some existing methods is introduced. Finally, based on the results obtained, the planned method has been determined to be the most feasible and successful for the MAGDM problem. Future research focuses on developing more decisionmaking methods, such as Einstein's hybrid AOs in the PFSS environment, using other operators. We are confident that these significant growths and prospects will help consider organizational research areas centered on the world's climate.

\section{Data Availability}

No data were used in this article.

\section{Conflicts of Interest}

The authors declare that they have no conflicts of interest.

\section{Acknowledgments}

The researchers would like to thank the Deanship of Scientific Research, Qassim University, for funding the publication of this project.

\section{References}

[1] L. A. Zadeh, "Fuzzy sets," Information and Control, vol. 8, no. 3, pp. 338-353, 1965.

[2] K. T. Atanassov, "Intuitionistic fuzzy sets," Fuzzy Sets and Systems, vol. 20, no. 1, pp. 87-96, 1986.

[3] Z. Zeshui $\mathrm{Xu}$, "Intuitionistic fuzzy aggregation operators," IEEE Transactions on Fuzzy Systems, vol. 15, no. 6, pp. 1179-1187, 2007.

[4] W. Wang and X. Liu, "Intuitionistic fuzzy geometric aggregation operators based on Einstein operations," International Journal of Intelligent Systems, vol. 26, no. 11, pp. 1049-1075, 2011.

[5] K. T. Atanassov, "Interval valued intuitionistic fuzzy sets," in Intuitionistic Fuzzy Sets, pp. 139-177, Physica, Heidelberg, Germany, 1999.

[6] R. R. Yager, "Pythagorean membership grades in multi-criteria decision making," IEEE Transactions on Fuzzy Systems, vol. 22, no. 4, pp. 958-965, 2013. 
[7] K. Rahman, A. Ali, M. Shakeel, M. A. Khan, and M. Ullah, "Pythagorean fuzzy weighted averaging aggregation operator and its application to decision making theory," The Nucleus, vol. 54, no. 3, pp. 190-196, 2017.

[8] K. Rahman, S. Abdullah, F. Husain, and M. S. A. Khan, "Approaches to Pythagorean fuzzy geometric aggregation operators," International Journal of Computer Science and Information Security, vol. 4, no. 9, pp. 174-200, 2016.

[9] L. Wang and N. Li, "Pythagorean fuzzy interaction power Bonferroni mean aggregation operators in multiple attribute decision making," International Journal of Intelligent Systems, vol. 35, no. 1, pp. 150-183, 2020.

[10] H. Garg, "Generalised Pythagorean fuzzy geometric interactive aggregation operators using Einstein operations and their application to decision making," Journal of Experimental and Theoretical Artificial Intelligence, vol. 30, no. 6, pp. 763-794, 2018.

[11] D. Molodtsov, "Soft set theory-first results," Computers and Mathematics with Applications, vol. 37, no. 4-5, pp. 19-31, 1999.

[12] P. K. Maji, R. Biswas, and A. R. Roy, "Soft set theory," Computers and Mathematics with Applications, vol. 45, no. 45, pp. 555-562, 2003.

[13] P. K. Maji, A. R. Roy, and R. Biswas, "An application of soft sets in a decision making problem," Computers and Mathematics with Applications, vol. 44, no. 8-9, pp. 1077-1083, 2002.

[14] P. K. Maji, R. Biswas, and A. Roy, "Intuitionistic fuzzy soft sets," Journal of Fuzzy Mathematics, vol. 9, pp. 677-692, 2001.

[15] R. M. Zulqarnain, X. L. Xin, M. Saqlain, and W. A. Khan, "TOPSIS method based on the correlation coefficient of interval-valued intuitionistic fuzzy soft sets and aggregation operators with their application in decision-making," Journal of Mathematics, vol. 2021, pp. 1-16, 2021.

[16] R. M. Zulqarnain, I. Siddique, R. Ali, D. Pamucar, D. Marinkovic, and D. Bozanic, "Robust aggregation operators for intuitionistic fuzzy hypersoft set with their application to solve MCDM problem," Entropy, vol. 23, no. 6, p. 688, 2021.

[17] R. Muhammad Zulqarnain, X. L. Xin, X. Long Xin, and M. Saeed, "Extension of TOPSIS method under intuitionistic fuzzy hypersoft environment based on correlation coefficient and aggregation operators to solve decision making problem," AIMS Mathematics, vol. 6, no. 3, pp. 2732-2755, 2021.

[18] H. Garg and R. Arora, "Generalized intuitionistic fuzzy soft power aggregation operator based on $\mathrm{t}$-norm and their application in multicriteria decision-making," International Journal of Intelligent Systems, vol. 34, no. 2, pp. 215-246, 2019.

[19] X. Peng, Y. Yang, and J. Song, "Pythagoren fuzzy soft set and its application," Computer Engineering, vol. 41, no. 7, pp. 224-229, 2015.

[20] T. M. Athira, S. J. John, and H. Garg, "A novel entropy measure of pythagorean fuzzy soft sets," AIMS Mathematics, vol. 5, no. 2, pp. 1050-1061, 2020.

[21] I. Siddique, R. M. Zulqarnain, R. Ali, A. Alburaikan, A. Iampan, and H. Abd El-Wahed Khalifa, "A decisionmaking approach based on score matrix for pythagorean fuzzy soft set," Computational Intelligence and Neuroscience, vol. 2021, pp. 1-16, Article ID 5447422, 2021.

[22] K. Naeem, M. Riaz, X. Peng, and D. Afzal, "Pythagorean fuzzy soft MCGDM methods based on TOPSIS, VIKOR and aggregation operators," Journal of Intelligent and Fuzzy Systems, vol. 37, no. 5, pp. 6937-6957, 2019.
[23] M. Riaz, K. Naeem, and D. Afzal, "Pythagorean m-polar fuzzy soft sets with TOPSIS method for MCGDM," Punjab University Journal of Mathematics, vol. 52, no. 3, pp. 21-46, 2020.

[24] R. M. Zulqarnain, I. Siddique, S. Ahmad et al., "Pythagorean fuzzy soft Einstein ordered weighted average operator in sustainable supplier selection problem," Mathematical Problems in Engineering, vol. 2021, Article ID 2559979, 16 pages, 2021.

[25] R. M. Zulqarnain, X. L. Xin, H. Garg, and W. A. Khan, "Aggregation operators of pythagorean fuzzy soft sets with their application for green supplier chain management," Journal of Intelligent and Fuzzy Systems, vol. 40, no. 3, pp. 5545-5563, 2021.

[26] R. M. Zulqarnain, X. L. Xin, I. Siddique, W. Asghar Khan, and M. A. Yousif, "TOPSIS method based on correlation coefficient under pythagorean fuzzy soft environment and its application towards green supply chain management," Sustainability, vol. 13, no. 4, p. 1642, 2021.

[27] R. M. Zulqarnain, X. L. Xin, H. Garg, and R. Ali, "Interaction aggregation operators to solve multi criteria decision making problem under pythagorean fuzzy soft environment," Journal of Intelligent and Fuzzy Systems, vol. 41, no. 1, pp. 1151-1171, 2021.

[28] H. Garg, "A new generalized Pythagorean fuzzy information aggregation using Einstein operations and its application to decision making," International Journal of Intelligent Systems, vol. 31, no. 9, pp. 886-920, 2016.

[29] H. Garg, "Generalized pythagorean fuzzy geometric aggregation operators using Einsteint-norm andt-Conorm for multicriteria decision-making process," International Journal of Intelligent Systems, vol. 32, no. 6, pp. 597-630, 2017.

[30] R. R. Yager, "Pythagorean fuzzy subsets," in Proceedings of the 2013 joint IFSA world congress and NAFIPS annual meeting (IFSA/NAFIPS), pp. 57-61, IEEE, Edmonton, AB, Canada, June 2013.

[31] K. Rahman, S. Abdullah, R. Ahmed, and M. Ullah, "Pythagorean fuzzy Einstein weighted geometric aggregation operator and their application to multiple attribute group decision making," Journal of Intelligent and Fuzzy Systems, vol. 33, no. 1, pp. 635-647, 2017.

[32] K. Rahman, S. Abdullah, A. Ali, and F. Amin, "Pythagorean fuzzy Einstein hybrid averaging aggregation operator and its application to multiple-attribute group decision making," Journal of Intelligent Systems, vol. 29, no. 1, pp. 736-752, 2020.

[33] R. Arora and H. Garg, "Robust aggregation operators for multi-criteria decision-making with intuitionistic fuzzy soft set environment," Scientia Iranica Transaction E Industrial Engineering, vol. 25, no. 2, pp. 931-942, 2018. 